\title{
Community structure of epibenthic megafauna in the Chukchi Sea
}

\author{
B. A. Bluhm ${ }^{1, *}$, K. Iken ${ }^{1}$, S. Mincks Hardy ${ }^{1}$, B. I. Sirenko ${ }^{2}$, B. A. Holladay ${ }^{1}$ \\ ${ }^{1}$ School of Fisheries and Ocean Sciences, 905 Koyukuk Dr., University of Alaska Fairbanks, Fairbanks, Alaska 99775, USA \\ ${ }^{2}$ Zoological Institute, Russian Academy of Sciences, Universitetskaja nab. 1, St. Petersburg, 199034 Russia
}

\begin{abstract}
Climate change and increased focus on resource development in Arctic Seas have fueled interest in the Chukchi Sea, yet few quantitative studies have been conducted on the larger, epifaunal component of seafloor communities, which serves important roles in sediment biogeochemical processes and provides a food source for fishes and marine mammals. Here we provide quantitative data on the present condition of benthic epifaunal abundance and biomass from the Chukchi shelf and examine the influence of environmental variables on epifaunal communities. We collected 45 beam trawl samples in the Russian and United States sectors of the Chukchi Sea in 2004, 2007 and 2008. Gross abundance estimates ranged from 229 to 70879 ind. $1000 \mathrm{~m}^{-2}$, and gross biomass estimates ranged from 1628 to $217023 \mathrm{~g}$ wet wt $1000 \mathrm{~m}^{-2}$. Overall, abundance and biomass were dominated by echinoderms (66 and $45 \%$, respectively) and crustaceans (17 and $31 \%$, respectively). The ophiuroid Ophiura sarsi and the snow crab Chionoecetes opilio overwhelmingly dominated abundance and biomass. The holothurian Myriotrochus rinkii also occurred in large numbers, and the urchin Strongylocentrotus pallidus was another major contributor to biomass. A total of 165 taxa (mostly species) were identified; the highest numbers were Mollusca (45) and Crustacea (33). Cluster analysis identified 6 distinct groups plus 6 unique stations with 54 to $88 \%$ between-cluster dissimilarity, with separation based largely on substrate type and latitude. Water mass characteristics and indices of food availability appeared less influential in generating the observed composition, abundance and biomass patterns. Comparisons with previous studies suggested an increase in overall epibenthic biomass since 1976, including an increase in the biomass of C. opilio.
\end{abstract}

KEY WORDS: Benthos $\cdot$ Epifauna $\cdot$ Chukchi Sea $\cdot$ Arctic $\cdot$ Community structure $\cdot$ Benthic-pelagic coupling $\cdot$ Temporal change $\cdot$ Chionoecetes opilio

\section{INTRODUCTION}

The Chukchi Sea, fed by Pacific waters through the Bering Strait, is the only Pacific gateway to the western Arctic proper. The inflow of nutrient-rich waters supports a productive marine ecosystem on parts of the Chukchi shelf (Coachman et al. 1975). Various ecosystem components, including the macrofaunal benthos, are relatively well-studied in this region (e.g. Grebmeier et al. 2006a). However, despite the significant change in sea-ice cover and other climate-forcing variables, the increased focus on resource development in the Chukchi Sea (Stroeve et al. 2005, Federal Register 2007), and the important role rich epifaunal communities play in carbon cycling on other Arctic shelves (Ambrose et al. 2001, Piepenburg et al. 1995, 2001), the epifaunal component of the megafauna in the Chukchi Sea ecosystem is poorly understood, with the exception of mollusks (Feder et al. 1994a). Here we present a relatively extensive quantitative dataset on epibenthic community structure, including sites throughout the United States and Russian sectors of the Chukchi shelf.

A significant portion of the seasonally ice-covered continental shelf of the Chukchi Sea under Bering Shelf Anadyr water is characterized by tight benthic-pelagic coupling due to shallow depths $(<150 \mathrm{~m})$ and the advection of nutrient-rich waters from the northern 
Bering Sea northward through the Bering Strait (Coachman et al. 1975, Springer \& McRoy 1993, Grebmeier et al. 2006a, Iken et al. in press). High primary productivity and rapid settlement of organic matter from the water column in these waters support high macrofaunal biomass, with peak values (25 to $60 \mathrm{~g} \mathrm{C} \mathrm{m}^{-2}$ ) being among the highest on the world's shallow shelves (Grebmeier et al. 1988, 2006a, Highsmith \& Coyle 1990, 1992, Sirenko \& Gagaev 2007). Phytoplankton and macrofaunal biomass levels are particularly high in the south-central Chukchi Sea, exceeding that of any other region on the Bering and southern Chukchi shelf (Walsh et al. 1989, Grebmeier 1993, Springer et al. 1996, Feder et al. 2007). Under the Alaska Coastal Water (ACW) in the eastern Chukchi Sea, benthic macrofaunal biomass is generally much lower $(<10 \mathrm{~g} \mathrm{C}$ $\mathrm{m}^{-2}$; Feder et al. 2007, Sirenko \& Gagaev 2007), and benthic coupling to the water column tends to be weaker (Dunton et al. 2005, Iken et al. in press). Few data exist for epifaunal abundance and biomass in this region, but, where they have been determined, values are high compared to those on other Arctic shelves (up to 53000 ind. $\mathrm{km}^{-2}$; $>2000 \mathrm{~kg}$ wet wt km-2 in the southeastern Chukchi Sea and Kotzebue Sound; Feder et al. 2005). These rich benthic communities serve as prey for a range of fishes, diving sea birds, and marine mammals (Feder \& Jewett 1981, Oliver et al. 1983, Coyle et al. 1997, Lovvorn et al. 2003).

Much of our knowledge regarding the ecology of the Chukchi Sea benthos comes from extensive studies of smaller macrofauna (e.g. Grebmeier et al. 2006a and references therein), whereas data for epibenthic megafauna are scarce. However, many of these largebodied epifaunal organisms contribute significantly to the overall benthic biomass on Arctic shelves, despite their patchy occurrence (Ambrose et al. 2001). Several groups are also highly mobile and play an important role in the redistribution and remineralization of the organic carbon reaching the seabed, in particular the often dominant echinoderms (Piepenburg \& Schmid 1996a,b, Piepenburg et al. 1995, 1996, 1997, 2001, Starmans et al. 1999, Piepenburg 2000, Sejr et al. 2000, Ambrose et al. 2001). The few quantitative studies on the epifauna in the Chukchi Sea have concentrated on the southeast (southeastern shelf extending into Kotzebue Sound; Feder et al. 2005) and inshore area of the northeastern region (Ambrose et al. 2001, Feder unpubl. data), including assessment of snow crab Chionoecetes opilio (Paul et al. 1997) and epifaunal mollusk populations (Feder et al. 1994a). Qualitative epifaunal information is available from near Point Hope around the Project Chariot site (Sparks \& Pereyra 1966) and from Point Barrow (MacGinitie 1955, 1959). Russian expeditions on the western Chukchi shelf have been conducted since the 20th century, but publications remain difficult to access, and trawl sampling was only qualitative in nature (Ushakov 1952). These information gaps are hindering attempts to quantify large-scale and/or long-term trends in epibenthic community structure, biomass and carbon flux in the face of increased human impacts such as oil and gas development (Federal Register 2007), rapid climatic change (Stroeve et al. 2005), and potential future fishing efforts (NPFMC 2009).

While biological continuous time-series data from the Chukchi Sea are not yet available, several recent studies have documented decadal-scale changes in biomass of various benthic components farther south in the northern Bering Sea, and trends appear to differ between size classes of organisms. Biomass of ampeliscid amphipods (Moore et al. 2003, Coyle et al. 2007), as well as total macrofaunal biomass (Dunton et al. 2005, Grebmeier et al. 2006b), declined roughly 50\% from the 1980 s to the early 2000 s. In contrast, significant increases in epibenthic abundance and biomass were recorded for the benthic community in Norton Sound from 1976 to 2002 (Hamazaki et al. 2005) and the southeastern Chukchi Sea from 1976 to 1998 (Fair \& Nelson 1999, Feder et al. 2005) and for ground fishes in the southeastern Bering Sea from 1960 to 2000 (Conners et al. 2002). In all cases, the changes have at least in part been attributed to North Pacific or Bering Sea regime shifts (e.g. Rodionov \& Overland 2005) and/or long-term climate change, but predation pressure imposed through selective bottom-feeding by marine mammals is another possible cause (Coyle et al. 2007). The goal of the present study was to provide quantitative data for epibenthic megafaunal abundance, biomass, and community structure for a large area of the Chukchi shelf and to examine relationships to environmental variables. Specifically, our main objectives were to: (1) quantify gross abundance, biomass, and diversity of epibenthic fauna (>4 mm), (2) identify environmental variables that correlate with epifaunal abundance and biomass, and (3) discuss potential temporal changes of Chukchi Sea epifauna with suggestions for monitoring strategies.

\section{MATERIALS AND METHODS}

The present study is part of the ongoing RussianAmerican Long-Term Census of the Arctic (RUSALCA) program launched with an interdisciplinary cruise in 2004 aboard the Russian vessel 'Professor Khromov'. The goal of this census is to gather long-term physical, chemical and biological data on the northern Bering and Chukchi Sea ecosystems to document their present conditions and to predict future change in this 
region. Additional sampling was conducted in 2009 and is also planned for 2012. Thus, the data presented here will enable us to compare future time-series data. In order to increase spatial coverage for this analysis, we also incorporated data collected with the same sampling methods at additional sites visited on 3 cruises in the United States sector of the Chukchi Sea (2007 to 2008) aboard the Japanese vessel 'Oshoro Maru' and the United States NOAA vessel 'Oscar Dyson' (Table 1).

Seventeen stations were sampled between 8 and 25 August 2004 at depths of from 39 to $54 \mathrm{~m}$ during RUSALCA (Fig. 1, Table 1, station designation 'R'). Six stations were sampled between 6 and 10 August 2007 at depths of from 25 to $50 \mathrm{~m}$ (stations ' $\mathrm{OM}^{\prime}$ ) and 7 stations were sampled between 4 and 14 September 2007 at depths of from 31 to $60 \mathrm{~m}$ (stations ' $\mathrm{OD}^{\prime}$ '). An additional 15 stations were sampled between 7 and 13 July 2008 at depths of from 36 to $51 \mathrm{~m}$ (stations 'OM-08') with the same gear but analyzed at lower taxonomic resolution. The entire region sampled covered an area

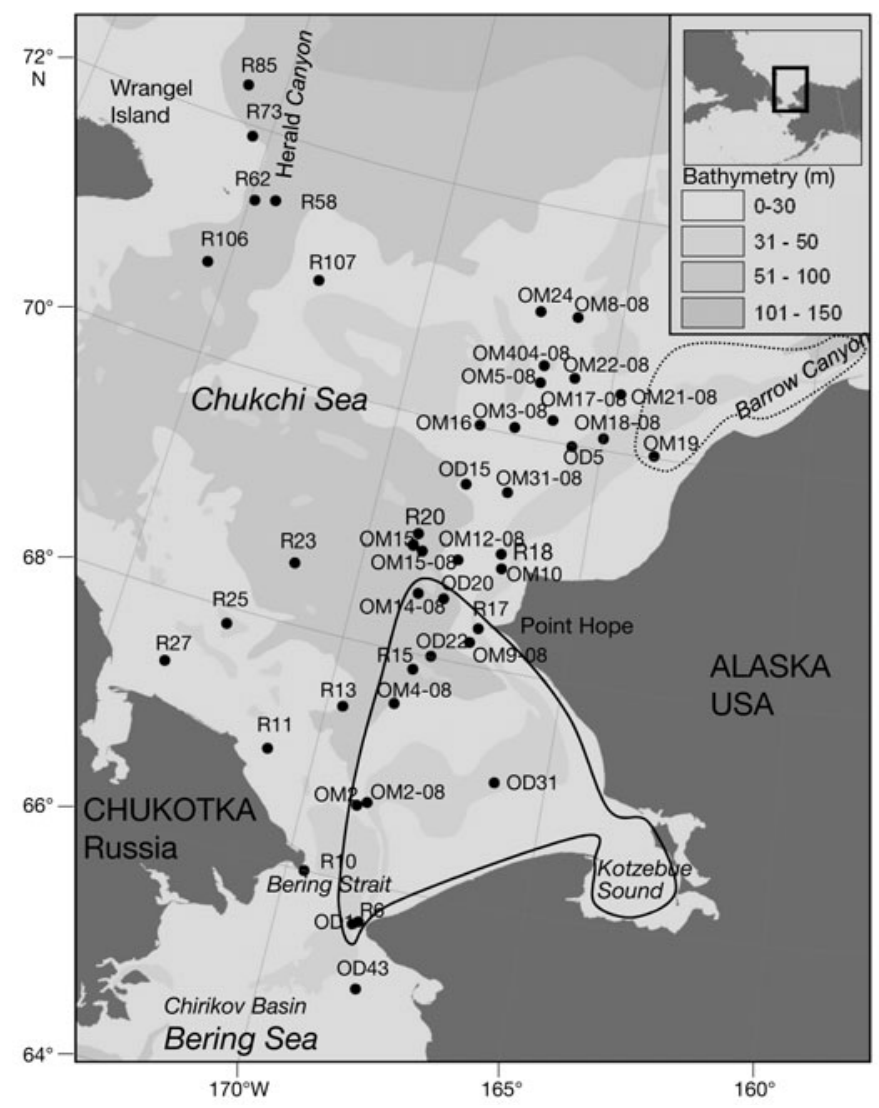

Fig. 1. Map of the study are with 45 sampling locations from 4 cruises marked. R: RUSALCA 2004; OM: Oshoru Maru 2007; OD: Oscar Dyson 2007; OM-08: Oshoru Maru 2008. Solid line shows the area studied by Feder et al. (2005); dotted line shows the area studied by Ambrose et al. (2001) from 65.12 to $72.32^{\circ} \mathrm{N}$ and from 163.71 to $175.99^{\circ} \mathrm{W}$. In summary, we sampled 44 stations in the Chukchi Sea and Bering Strait, and 1 station in the northern Bering Sea during 4 expeditions from 2004 to 2008 (Fig. 1, Table 1).

Study area. The Chukchi Sea is a shallow shelf sea ( $\sim 50 \mathrm{~m}$ average depth) that extends $\sim 800 \mathrm{~km}$ northward from the Bering Strait to the shelf break at the $200 \mathrm{~m}$ isobath. Two canyons incise the shelf in the north, of which we sampled Herald Canyon in the northwestern Chukchi Sea (Fig. 1). Sediments are mostly muddy on the outer shelf and coarser, with sand and gravel, on the inner shelf and at locations with relatively high currents such as the Bering Strait (www. ngdc.noaa.gov/mgg/geology/size.html; Naidu 1988).

The mean flow over much of the shelf is northward due to the Pacific-Arctic pressure gradient (Winsor \& Chapman 2004, Weingartner et al. 2005). The study area is influenced by 3 dominant water masses (Coachman 1987, Walsh et al. 2005, Weingartner et al. 2005). ACW in the east is characterized by low productivity and nutrient concentrations, warm surface temperatures, and low salinities $(\leq 31.9)$. Anadyr water $(\mathrm{AW})$ in the western Chukchi Sea is characterized by high primary productivity and nutrient concentrations and colder, more saline waters $(\geq 32.5)$. Bering Shelf Water (BSW), which is relatively high in nutrients, particulate organic carbon concentrations, and primary productivity, is located between these 2 water masses, and has salinities between 31.9 and 32.5. AW and BSW mix, are often referred to as Bering Shelf Anadyr water (BSAW) (salinity $>31.9$; Feder et al. 2005, Grebmeier et al. 2006a), and are combined as such in our analysis below. Stn R27 (Fig. 1) had the strong freshwater signal of a coastal lagoon (Pickart 2006), and the location may, at times, be located under the Siberian Coastal Current (Weingartner et al. 1999). During the RUSALCA cruise, a mass of cold and salty northward flowing winter water (WW), apparently formed from a reservoir of winter water in the area southeast of Wrangel Island and from water advected through the Bering Strait, remained in part of Herald Canyon from the previous winter (Pickart et al. 2005, in press).

Trawl collections. A plumb staff beam trawl (after Gunderson \& Ellis 1986) with a $2.26 \mathrm{~m}$ effective opening and a net mesh of $7 \mathrm{~mm}$, with a $4 \mathrm{~mm}$ cod end liner, was used for epibenthic collections. Tow duration ranged from 1 to $6 \mathrm{~min}$, area swept ranged from 107 to $1257 \mathrm{~m}^{2}$ and towing speed was approximately 1.5 knots. Wire was deployed at a rate of $3.5 \mathrm{~m} \mathrm{~m}^{-1}$ of water depth; details can be found in Norcross et al. (in press). Tows at Stns R10, R11 and R15 were not quantitative because of unknown area covered, but were included for determination of relative composition and number of taxa. 


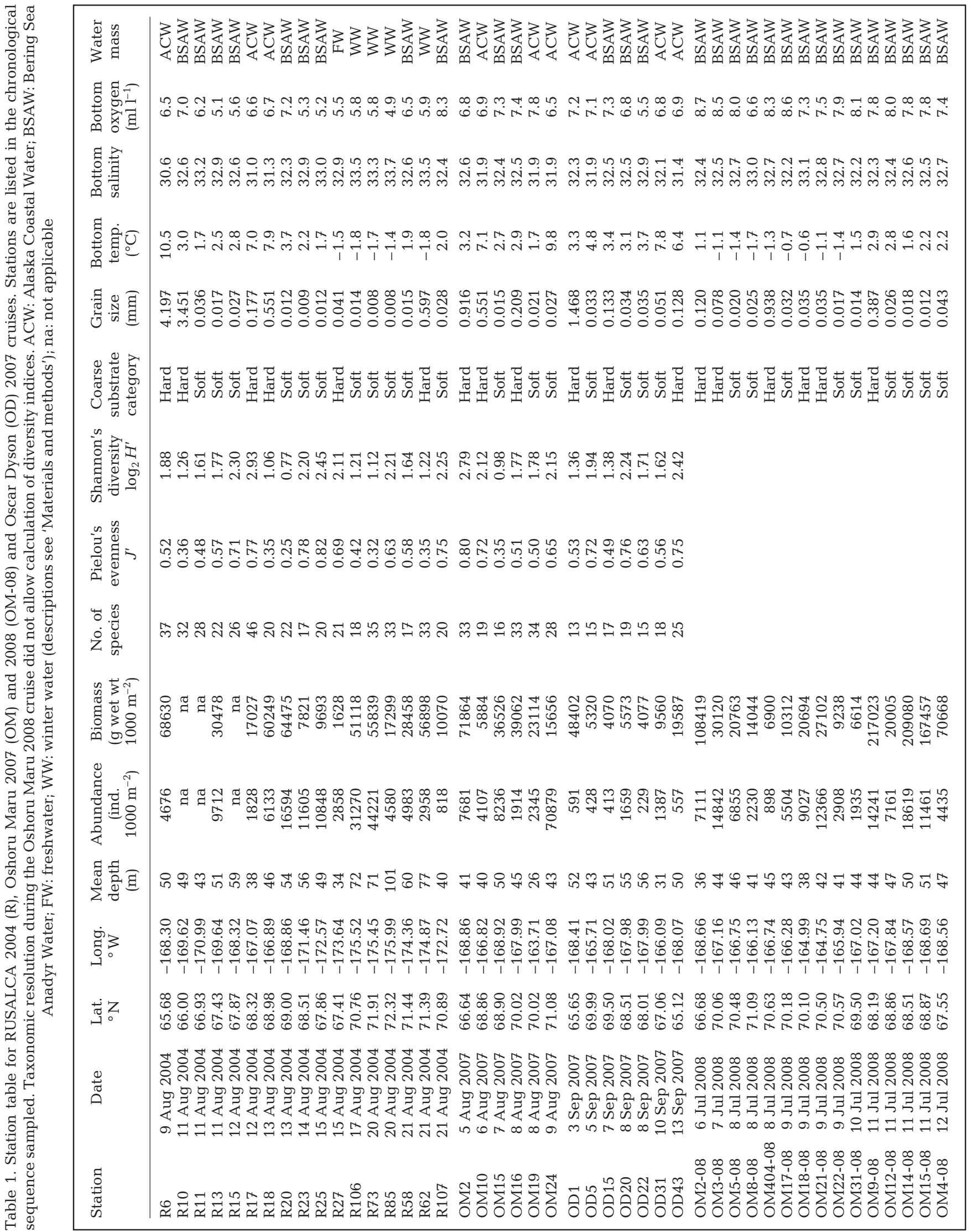


Epibenthic invertebrates from trawl catches were washed on deck and sorted to the lowest possible taxonomic level, in most cases species level. Wet weight for each taxon was determined on deck using spring scales, and numbers of individuals were noted. For colonial organisms (sponges, colonial ascidians, bryozoans, hydrozoans), only wet weights were recorded. Voucher specimens from each taxon were preserved in $4 \%$ buffered formalin and later transferred to $50 \%$ isopropanol for long-term storage. Biomass and abundance were estimated from area swept (= net swath $\times$ distance towed) and normalized to $1000 \mathrm{~m}^{-2}$. These commonly used estimates are considered semi-quantitative because of variable trawl performance across bottom types, net avoidance behavior of some taxa and difficulties in precisely weighting the gear to obtain ideal bottom contact; hence, we refer to these data as gross estimates (Eleftherious \& MacIntyre 2005). However, alternative sampling gear (e.g. grabs and corers) sample much smaller areas and thus do not effectively sample the larger and/or rarer epifaunal taxa. Epifaunal assessments are ideally conducted using a combination of trawls and photographic surveys (Eleftherious \& MacIntyre 2005), but photographic equipment was not available for use in the present study.

Taxonomic identifications were conducted by the authors and the taxonomists listed in Appendix 1. Upon publication, detailed taxonomic information will be made available through the Ocean Biogeographic Information System and the Global Biodiversity Information Facility.

Data analysis. All individuals were included in data analysis, whether identified to species or higher taxonomic units; colonial organisms were excluded in abundance-based analyses. Spatial patterns in abundance, biomass and number of taxa (see Figs. 2 to 5) were produced using ArcGIS v.9.1 (ESRI 2005), with bin sizes determined according to the Jenks' natural breaks classification scheme. This scheme selects breaks, relatively large jumps in the data values, in the ordered distribution of values that minimize the within-class sum of squared differences. Detailed community composition analysis was performed for the combined datasets of the 2004 and 2007 cruises, using multivariate statistics of the software package PRIMER v.6 (Clarke \& Gorley 2006). Collections from the 2008 cruise were excluded from the community analysis due to low taxonomic resolution. The 2008 data were included in figures showing total abundance, biomass and relative faunal composition, as well as snow crab Chionoecetes opilio biomass, and in statistical tests on the relationship of total abundance and biomass to environmental variables (see Figs. 2 to 4). Bray-Curtis similarity was calculated for the fourth-root-transformed biomass matrix rather than for the abundance matrix because abundance cannot be recorded for colonial taxa. In some cases, taxon groups were used for community analysis (e.g. Argis lar and Neocrangon communis; see Table 2) because they were not consistently separated on the species level during the initial trawl sorting. Non-metric multi-dimensional scaling (NMDS) was used to visualize the resulting patterns in similarity between stations. Species contributing most to the similarities of stations within and between clusters were identified using the Similarity of Percentages routine (SIMPER) in PRIMER. Diversity indices (Pielou's evenness, $J^{\prime}$; Shannon's diversity, $\log _{2} H^{\prime}$ ) were calculated using the DIVERSE routine in PRIMER.

We compiled a set of environmental data for each station, including several variables related to water masses (bottom temperature, bottom salinity, oxygen concentration), sediment characteristics (substrate category, mean grain size), and indicators of food supply (integrated chlorophyll concentrations, macrofaunal biomass, sediment organic carbon content), as well as variables with (potentially) indirect relationships to community structure (latitude, water depth). All variables were chosen on the basis of their strong structuring effects on Arctic benthic communities (temperature: Mayer \& Piepenburg 1996, Conlan et al. 2008; salinity: Feder et al. 1994a, Mayer \& Piepenburg 1996, Deubel et al. 2003; oxygen: Deubel 2000, Conlan et al. 2008; grain size: Feder et al. 1994a; coarse substrate: Feder et al. 1994b, Mayer \& Piepenburg 1996; primary production: MacDonald et al. in press; depth: Deubel 2000, Conlan et al. 2008, MacDonald et al. in press; latitude: Deubel 2000). Relationships between the bulk parameters abundance and biomass and several of the environmental variables were tested with Student's $t$-tests, ANOVA and Pearson correlations using SYSTAT software. Analysis of similarity (ANOSIM) was used to provide statistical tests of between-group similarity for stations grouped by substrate category and water mass, with global $\mathrm{R}=1$ indicating groups with completely distinct communities and global $\mathrm{R}=0$ indicating complete overlap in community composition between groups (Clarke \& Warwick 2001). The entire normalized environmental matrix was correlated with the entire epifaunal species matrix based on biomass (excluding 2008 data) using the BIO-ENV procedure in PRIMER (Clarke \& Gorley 2006).

Substrate category (hard/soft) was assigned based on visual inspection of trawls and grabs, with 'hard' assigned to stations with substantial amounts of gravel and/or cobble and often shell hash and 'soft' assigned to stations with mud and sand and no or little gravel and cobble. Mean sediment grain sizes (mm) were obtained from the Seafloor Sediment Grain Size Database NGDC Data Set G00127 (www.ngdc.noaa.gov/ 
mgg/geology/size.html) from locations near the sampling stations, because this information was not collected during all cruises reported here. These data were compiled from multiple contributors and collected during various expeditions over several decades (1950s to 2000s) using a variety of sampling tools; they should thus be considered with some caution. However, when these data were compared with grain size values determined on the basis of our RUSALCA 2004 samples (J. Grebmeier and L. Cooper, Univ. Maryland, unpubl. data), they matched reasonably well.

Bottom water temperature, salinity and oxygen concentrations (Table 1) were obtained from CTD casts performed concurrently at each sampling station by collaborating groups and were provided by Dr. R. Pickart (unpubl. data; Woods Hole Oceanographic Institution, RUSALCA cruise), the Oshoru Maru website (http://odyssey.fish.hokudai.ac.jp/IPY/) and Dr. L. Eisner (unpubl. data; NOAA Alaska Fisheries Science Center, Oscar Dyson cruise). Water masses were assigned based on bottom salinity values after Coachman (1987). Water column-integrated chlorophyll data were obtained from Lee et al. (2007; RUSALCA cruise), the Oshoru Maru website (http://odyssey.fish.hokudai.
ac.jp/IPY/) and Dr. L. Eisner (unpubl. data; NOAA Alaska Fisheries Science Center, Oscar Dyson cruise).

Macrofaunal biomass data for RUSALCA stations were provided by J. Grebmeier and L. Cooper (unpubl. data; University of Maryland). Additional macrofaunal biomass and sediment organic content data were taken from Grebmeier et al. (2006a), using the midpoint of the bins in their Figs. 4 and 5, respectively. To test a potential relationship between trawl-collected epibenthic megafauna and macrofaunal biomass determined from grab samples, we compiled a dataset of paired measurements for these 2 size classes using macrofaunal biomass from Grebmeier and Cooper (unpubl. data) and Grebmeier et al. (2006a) and epifaunal biomass from the present study, from the same locations. Additionally, a subset of epifaunal data from Feder et al. (2005) was paired with a subset of macrofaunal data from Feder et al. (2007), using only locations that were very close to each other. A total of 61 locations were considered. We also conducted a preliminary comparison of epifaunal biomass and community composition at 8 stations in the southeastern Chukchi Sea that were sampled in 1976 (Feder et al. 2005; their Fig. 1), as well as in the present study.
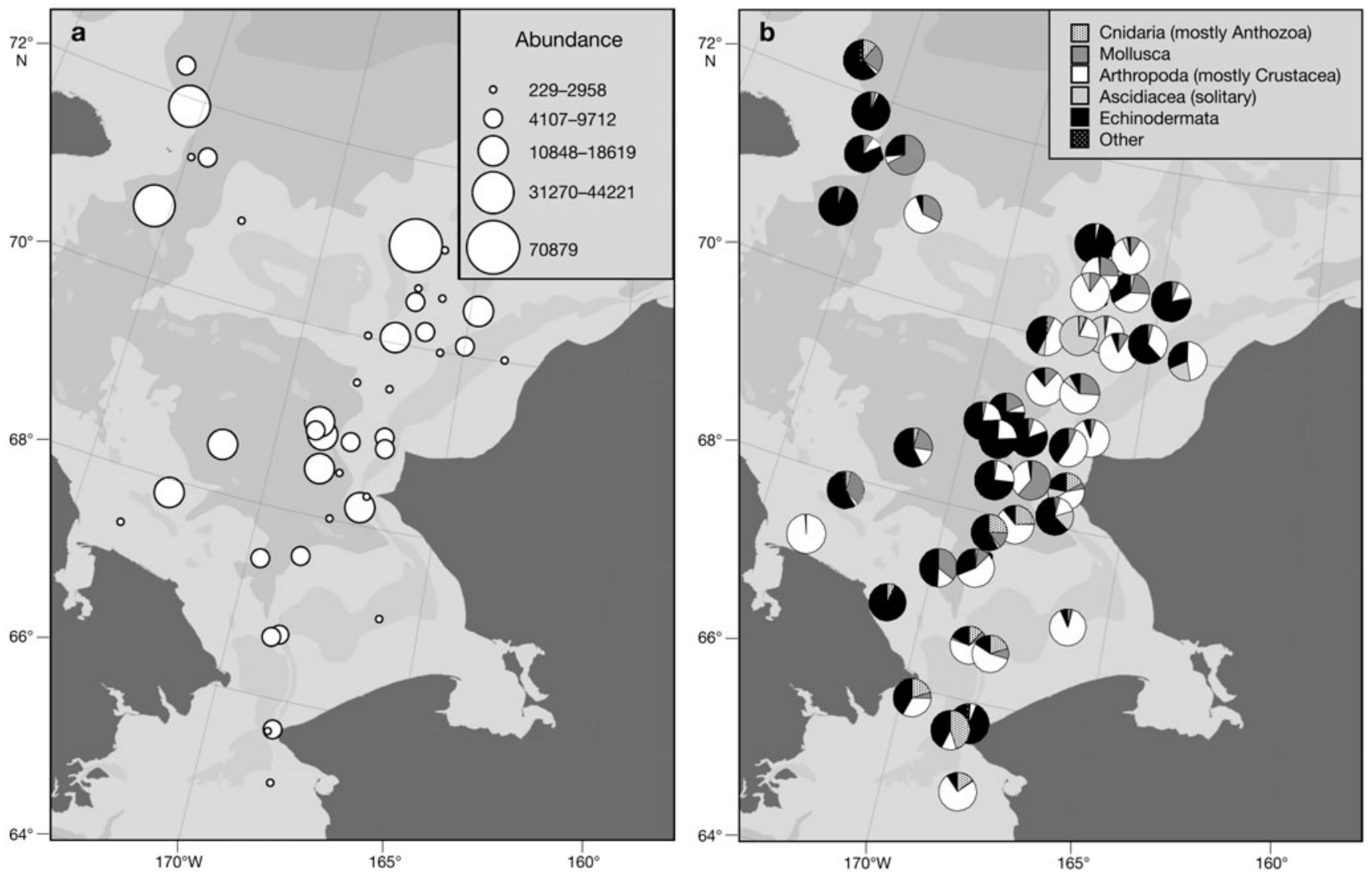

Fig. 2. Epifaunal abundance. (a) Absolute abundance (ind. $1000 \mathrm{~m}^{-2}$ ). Bins delineated according to Jenks' natural breaks criteria (see 'Materials and methods'). (b) Relative composition 


\section{RESULTS}

\section{Distribution, abundance and biomass}

Gross abundance ranged from 229 ind. $1000 \mathrm{~m}^{-2}$ at Stn OD22 to 70879 ind. $1000 \mathrm{~m}^{-2}$ at Stn OM24 (mean \pm SD across all stations $8736 \pm 12647$ ind. $1000 \mathrm{~m}^{-2}$ ) (Table 1, Fig. 2a). There was no discernable latitudinal or longitudinal trend in abundance, although stations with particularly high abundance were farthest north. Gross biomass estimates ranged from $1628 \mathrm{~g}$ wet wt $\mathrm{m}^{-2}$ at Stn R27 to 217023 g wet wt $\mathrm{m}^{-2}$ at Stn OM9-08 (mean \pm SD across all stations $38863 \pm 49418 \mathrm{~g}$ wet $\mathrm{wt}^{-2}$ ) (Fig. 3a). Biomass values were generally highest in the area from the Bering Strait to an area northwest of Point Hope and in Herald Canyon.

Abundance and biomass were dominated by echinoderms at many stations ( $67 \%$ of mean abundance and $45 \%$ of mean biomass across all stations). The group was particularly prevalent in the Herald Canyon area and the Bering Strait in both abundance and biomass, and also numerically at most stations between about 67 and $69^{\circ} \mathrm{N}$ (Fig. 2b). Echinoderm biomass was also dominant at many central stations (Fig. 3b). Crusta- ceans contributed most to abundance and biomass at some of the southeastern and northeastern stations. The group made up $18 \%$ of the mean abundance and $31 \%$ of the mean biomass in the study area and, numerically, $90 \%$ of the crustaceans at Stn R27, where amphipods overwhelmingly dominated. Overall, mollusks contributed $8 \%$ to mean abundance and $9 \%$ to mean biomass in the study area. Stns R58 (Herald Canyon) and OD20 (central Chukchi) were numerically dominated by mollusks, mostly gastropods (68 and $61 \%$, respectively), and, at Stns R15 and R25 (southwestern Chukchi), mollusks dominated biomass (42 and $36 \%$, respectively, of station biomass). Ascidians comprised $5 \%$ of the overall mean abundance and $8 \%$ of the mean biomass in the study area. Stns OM17-08 and OM3-08 (northeastern Chukchi) were numerically dominated by solitary ascidians (49 and $72 \%$, respectively), and, at Stn OM9-08 (near Pt. Hope), ascidians contributed most to biomass (51\%; majority of the group 'other'). Cnidarians (mostly Actinaria) did not dominate abundance or biomass at any station, but made up about $3 \%$ of the mean abundance and $4 \%$ of the mean biomass in the study area, while sponges and bryozoans each contributed $<1 \%$ to total biomass.
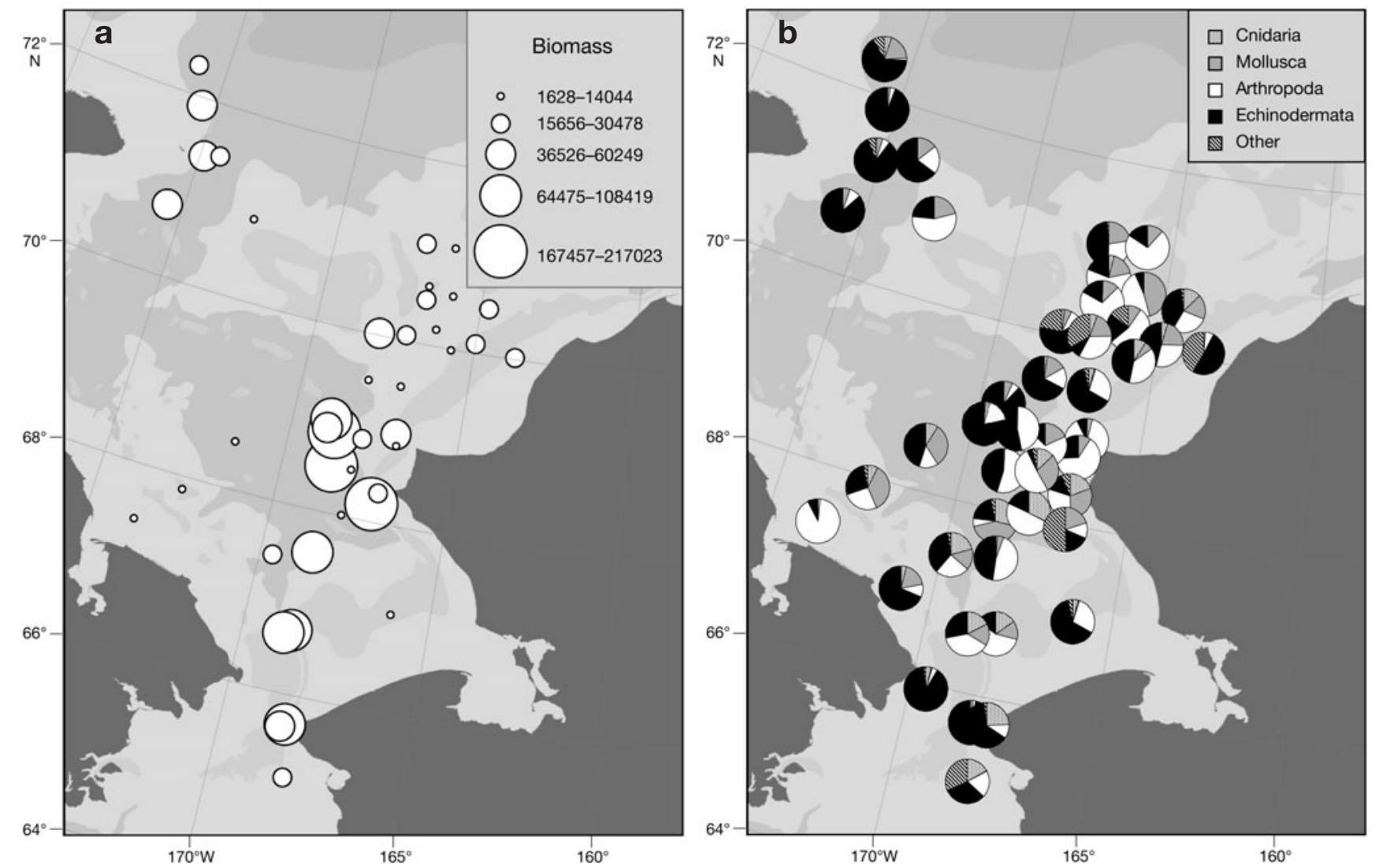

Fig. 3. Epifaunal biomass. (a) Absolute biomass (g wet wt $1000 \mathrm{~m}^{-2}$ ). Bins delineated according to Jenks' natural breaks criteria (see 'Materials and methods'). (b) Relative composition 
The vast majority (>70\%) of all individuals collected in all trawls combined consisted of only 3 species: the brittle star Ophiura sarsi $(60.0 \%)$, the sea cucumber Myriotrochus rinkii (8.6\%) and the snow crab Chionoecetes opilio $(4.8 \%)$. Similarly, only 3 species contributed $>10 \%$ each to the total biomass in the sampling area: O. sarsi $(16.9 \%)$, the sea urchin Strongylocentrotus pallidus $(14.1 \%)$ and C. opilio $(13.0 \%)$. The abundance of $C$. opilio, a commercially valuable species in the Bering Sea and northwestern Atlantic, ranged from 0 (at Stn OD1) to 4017 ind. $1000 \mathrm{~m}^{-2}$ at St OM14-08 (mean \pm SD across all stations: $765 \pm 1103$ ). The contribution of $C$. opilio abundance to total abundance reached a maximum of 67\% at Stn OM404-08. C. opilio biomass ranged from 0 at Stn OD1 to 111552 $\mathrm{g}$ wet wt $1000 \mathrm{~m}^{-2}$ at Stn OM5 (mean \pm SD across all stations $10135 \pm 22$ 052) and was highest in the southcentral and southeastern Chukchi Sea (Fig. 4). The relative contribution of $C$. opilio biomass to total biomass reached a maximum of $69 \%$ at Stn OM404-08 (mean \pm SD: $20 \pm 18 \%$ ).

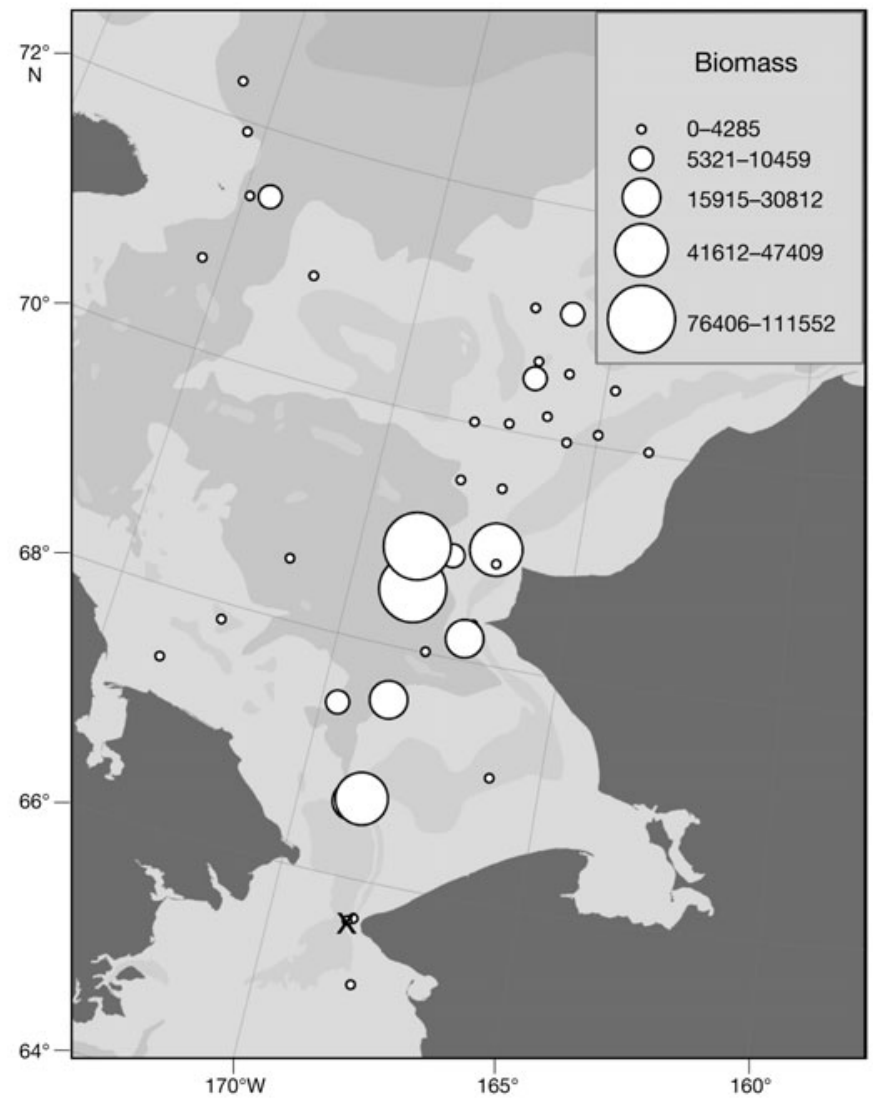

Fig. 4. Chionoecetes opilio. Biomass (g wet wt $1000 \mathrm{~m}^{-2}$ ) of this commercially harvested species in the Bering Sea. Bins delineated according to Jenks' natural breaks criteria (see 'Materials and methods'). The cross marks the only sampling location (OD1 in the Bering Strait) where no snow crabs were caught

\section{Taxon numbers and diversity}

The total number of taxa was 165, 10 of which were taxonomically only identified to a family or higher level, while all others were considered individual species or putative species (see Appendix 1). Across the whole study area, mollusks had the highest number of taxa (45), followed by crustaceans (33), bryozoans (23), echinoderms (23), ascidians (14), cnidarians (13) and sponges (8). The numbers of taxa were highest in the Bering Strait and Herald Canyon areas, and at stations near the Alaskan coast (Fig. 5a). The maximum number of taxa was 47 at Stn R17 (Point Hope), and the lowest number was 13 at Stn OD22, about 35 $\mathrm{km}$ away. Gastropods contributed most to the total number of taxa at soft-bottom stations, whereas colonial taxa (Porifera, Bryozoa and Ascidia) contributed substantially to the total number of taxa at hardbottom sites (Fig. 5b). Neither the Shannon diversity index (range from 0.77 at Stn R20 to 2.93 at Stn R17), nor Pielou's evenness (range from 0.25 at Stn R20 to 0.82 at Stn R25; Table 1) showed any discernable spatial trends.

\section{Station similarity}

Cluster analysis of fourth-root-transformed biomass data resulted in 6 significant station clusters and 6 unique stations not included in any of the significant clusters ( $p=0.05$, Similarity Profile test in PRIMER). Bray-Curtis similarity within clusters was from 53 to $71 \%$, except for 1 cluster at only $34 \%$ similarity (Table 2, Fig. 6a). Dissimilarity between pairs of clusters ranged from 54 to $88 \%$ (Table 3). Mostly, clusters included stations in a connected geographic area (Fig. 6a). Ordination of stations in the NMDS (Fig. 6b) showed little overlap between stations from different clusters, with the exception of R11 and OD31. Three of the unique stations identified using fourth-roottransformations were part of multi-station clusters using square-root-transformations in the cluster analysis; OM19 grouped with OD31, OD22 grouped with the central \& north (CN) cluster, and R62 grouped with the south/southeast (SSE) cluster (not shown). We found no significant difference using 'cruise' as a factor (ANOSIM, global $\mathrm{R}=0.062, \mathrm{p}=0.208$ ), indicating that station clusters were not the result of investigator- or method-related bias between the 3 cruises.

Four to 6 taxa, mostly within echinoderms, crustaceans and mollusks, contributed $\sim 50 \%$ of the similarity within each of the 6 main station clusters (Table 2), while 15 or more species contributed $50 \%$ to the dissimilarity between clusters, of which the 3 dominant taxa are given for each cluster in Table 3 . The 5 bio- 
mass-dominant taxa per cluster were mostly echinoderms and crustaceans, but also included several actinarians and gastropods, all of which contributed $\sim 60$ to $>90 \%$ to the total biomass in each cluster (Table 4). Most of the dominant species had a broad latitudinal distribution across the study area (Fig. 7). Exceptions included the sea star Lethasterias nanimensis and the anemone Cribrinopsis sp., which only occurred in the southern Chukchi Sea, and the sea stars Ctenodiscus crispatus and Urasterias linkii, which only occurred at the northern stations in Herald Canyon.

\section{Environmental and biological linkages}

The combination of environmental variables that best matched the biological matrix (excluding OM-08 stations) included 7 variables (water depth, latitude, substrate category, grain size, bottom temperature, oxygen and sediment organic content) with a moderately high correlation coefficient of 0.533 (BIO-ENV; Table 5). The combination of latitude and substrate yielded a correlation coefficient of 0.535, which increased very little with the addition of other variables.

When correlated to the variable 'substrate category', station groups 'soft' and 'hard' were significantly different with substantial separation (ANOSIM, global $\mathrm{R}=0.42, \mathrm{p}=0.001$ ). Station similarity based on water masses had a low global $\mathrm{R}=0.21$ (ANOSIM, $\mathrm{p}=0.011$ ), suggesting little separation. Pairwise tests indicated no difference in community structure between stations in the 2 main water masses in the region, ACW and BSAW ( $R=0.05, p>0.05)$. Differences were larger and significant between ACW and WW $(\mathrm{R}=0.41, \mathrm{p}=$ 0.019).

Overall, bulk abundance and biomass were not significantly different at hard- versus soft-bottom stations ( $p=0.479$ and $p=0.099$, respectively, $t$-test), between different water masses $(\mathrm{p}=0.663$ and $\mathrm{p}=0.263$, respectively, ANOVA), or between different station clusters identified from cluster analysis $(\mathrm{p}=0.285$ and $\mathrm{p}=0.497$, respectively, ANOVA). When sessile (Porifera, Cnidaria, Bivalvia, Cirripedia, Ascidiacea, Brachiopoda, Bryozoa) and mobile fauna (other fauna) were analyzed separately, results were similar, except
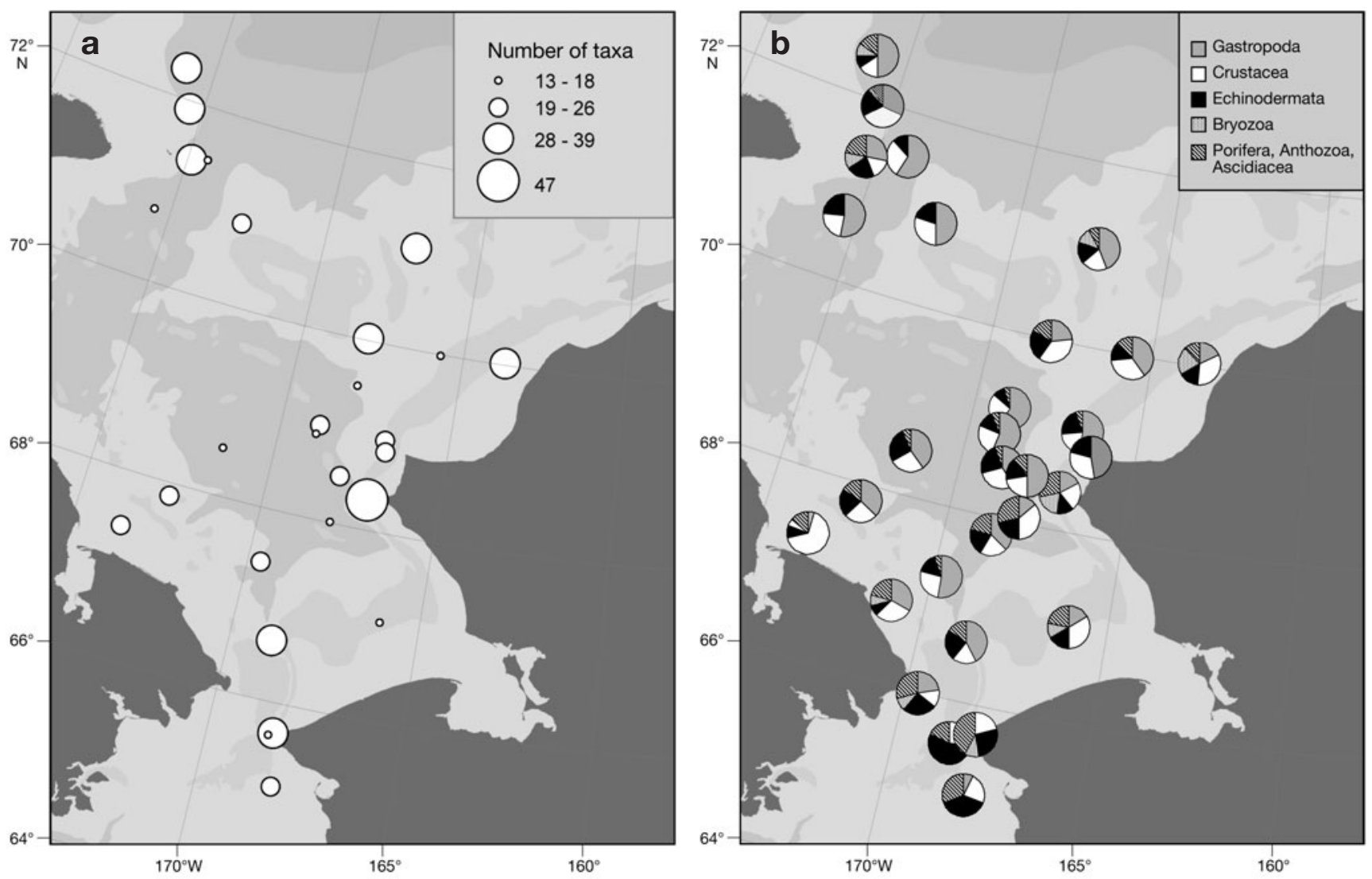

Fig. 5. Number of epifaunal taxa in the Chukchi Sea. (a) Absolute numbers of taxa. Bins delineated according to Jenks' natural breaks criteria (see 'Materials and methods'). (b) Relative taxonomic composition. Note: 2008 data were excluded 
Table 2. Epifaunal species contributing approximately $50 \%$ of the similarity within each of the 6 main cluster groups identified from hierarchical clustering. Sim.: similarity; SD: standard deviation; Contrib.: contribution; Cum.: cumulative

\begin{tabular}{|c|c|c|c|c|c|}
\hline & $\begin{array}{l}\text { Av. biomass } \\
\text { (g wet wt } \\
100 \mathrm{~m}^{-2} \text { ) }\end{array}$ & $\begin{array}{c}\text { Av. } \\
\text { similarity } \\
(\%)\end{array}$ & $\begin{array}{l}\text { SD } \\
\text { of } \\
\text { Sim. }\end{array}$ & $\begin{array}{c}\text { Contrib. } \\
(\%)\end{array}$ & $\underset{(\%)}{\text { Cum. }}$ \\
\hline \multicolumn{6}{|l|}{$\begin{array}{l}\text { Group south/southeast (SSE), } \\
\text { average similarity: } 34 \%\end{array}$} \\
\hline Strongylocentrotus pallidus & 6.9 & 3.4 & 1.0 & 10.2 & 10.2 \\
\hline Leptasterias groenlandica & 5.0 & 3.0 & 2.6 & 9.1 & 19.3 \\
\hline Chionoecetes opilio & 4.7 & 2.8 & 1.4 & 8.2 & 27.5 \\
\hline Hyas coarctatus & 5.1 & 2.6 & 1.4 & 7.8 & 35.3 \\
\hline Argis lar \& Neocrangon communis & 3.9 & 2.4 & 1.4 & 7.2 & 42.5 \\
\hline Gersemia sp. (cf. rubiformis) & 4.7 & 2.3 & 1.0 & 6.9 & 49.5 \\
\hline \multicolumn{6}{|l|}{$\begin{array}{l}\text { Group southwest (SW), } \\
\text { average similarity: } 55 \%\end{array}$} \\
\hline Myriotrochus rinkii & 9.9 & 10.9 & & 20.0 & 20.0 \\
\hline Henricia tumida & 6.3 & 6.9 & & 12.7 & 32.6 \\
\hline Chionoecetes opilio & 7.1 & 5.4 & & 9.8 & 42.5 \\
\hline Cribrinopsis/Epiactis sp. & 6.6 & 4.7 & & 8.6 & 51.0 \\
\hline \multicolumn{6}{|l|}{$\begin{array}{l}\text { Group south-central-west (SCW), } \\
\text { average similarity: } 58 \%\end{array}$} \\
\hline Urticina sp.? & 5.5 & 6.0 & 8.9 & 10.3 & 10.3 \\
\hline Chionoecetes opilio & 5.2 & 5.8 & 7.1 & 9.9 & 20.3 \\
\hline Cryptonatica affinis & 5.5 & 5.7 & 4.1 & 9.8 & 30.1 \\
\hline Lunatia pallida & 4.5 & 4.9 & 59.3 & 8.5 & 38.5 \\
\hline Myriotrochus rinkii & 4.4 & 4.4 & 3.5 & 7.6 & 46.1 \\
\hline Amphiodia craterodmeta & 5.0 & 4.3 & 1.2 & 7.4 & 53.5 \\
\hline \multicolumn{6}{|l|}{$\begin{array}{l}\text { Group central \& north }(\mathrm{CN}) \text {, } \\
\text { average similarity: } 53 \%\end{array}$} \\
\hline Chionoecetes opilio & 7.8 & 8.2 & 7.8 & 15.5 & 15.5 \\
\hline Pagurus rathbuni & 6.3 & 6.3 & 5.5 & 11.9 & 27.4 \\
\hline Leptasterias polaris & 5.2 & 5.6 & 3.9 & 10.6 & 38.0 \\
\hline Argis lar \& Neocrangon communis & 4.7 & 5.4 & 5.5 & 10.2 & 48.2 \\
\hline Buccinum elatior & 4.5 & 4.7 & 5.9 & 9.0 & 57.1 \\
\hline \multicolumn{6}{|l|}{$\begin{array}{l}\text { Group Herald Canyon area (HC), } \\
\text { average similarity: } 63 \%\end{array}$} \\
\hline Ctenodiscus crispatus & 10.8 & 12.9 & & 20.2 & 20.2 \\
\hline Chionoecetes opilio & 8.2 & 9.9 & & 15.6 & 35.9 \\
\hline Tachyrhynchus erosus & 5.9 & 6.6 & & 10.5 & 46.3 \\
\hline Pagurus rathbuni & 4.3 & 5.4 & & 8.5 & 54.8 \\
\hline \multicolumn{6}{|l|}{$\begin{array}{l}\text { Group central-east (CE), } \\
\text { average similarity: } 71 \%\end{array}$} \\
\hline Gorgonocephalus caryi & 6.1 & 9.5 & & 13.4 & 13.4 \\
\hline Chionoecetes opilio & 5.1 & 8.2 & & 11.6 & 25.0 \\
\hline Neptunea ventricosa & 4.1 & 6.9 & & 9.7 & 34.7 \\
\hline Argis lar \& Neocrangon communis & 3.7 & 6.5 & & 9.1 & 43.8 \\
\hline Pagurus rathbuni & 3.7 & 5.5 & & 7.7 & 51.5 \\
\hline
\end{tabular}

that sessile biomass was significantly different between the clusters SSE and $\mathrm{CN}, \mathrm{CN}$ and unique Stn OM19, and Herald Canyon and unique Stn OM19 (ANOVA $p=0.014$ ). Epifaunal biomass was not significantly correlated with sediment grain size (Pearson correlation coefficient $=0.126, p=0.426$, Bartlett's chisquare $=0.634, \mathrm{n}=42$ ), number of taxa (Pearson correlation coefficient $=0.367, p=0.060$, Barlett's chi-square
$=3.547, \mathrm{n}=27$ ), sediment organic carbon content (Pearson correlation coefficient $=0.194, \mathrm{p}=0.130$, Barlett's chisquare $=2.288, \mathrm{n}=42$ ), integrated chlorophyll concentration (Pearson correlation coefficient $=0.138, \mathrm{p}=0.49$, Bartlett's chi-square $=0.47, \mathrm{n}=27$ ), bottom temperature (Pearson correlation coefficient $=0.021, \mathrm{p}=0.897$, Bartlett's chi-square $=0.017, \mathrm{n}=42$ ), bottom salinity (Pearson correlation coefficient $=-0.027, \mathrm{p}=0.865$, Bartlett's chi-square $=0.029, \mathrm{n}=42$ ), or bottom oxygen concentration (Pearson correlation coefficient $=0.231, \mathrm{p}=0.141$, Bartlett's chisquare $=2.164, \mathrm{n}=42$ ). Analyzing sessile and mobile biomass separately yielded the same result (i.e. no significant correlations with the above-listed variables), except that sessile biomass alone was correlated with the number of taxa (Pearson correlation coefficient $=0.632, \mathrm{p}<0.001$, Barlett's chi-square $=$ 14.013, $\mathrm{n}=27$ ).

The number of taxa was not significantly different between hard-bottom (mean \pm SD: $27.4 \pm 9.7$ ) and soft-bottom stations (mean $\pm \mathrm{SD}: 22.4 \pm 6.7 ; \mathrm{p}=$ 0.115 , $t$-test, $\mathrm{n}=30)$, clusters $(\mathrm{p}=0.127$, ANOVA, $\mathrm{n}=30$ ), or water masses ( $\mathrm{p}=$ 0.546, ANOVA, $\mathrm{n}=30$ ). ShannonWiener's $H^{\prime}$ was virtually the same at hard-bottom (mean \pm SD: $1.84 \pm 0.60$ ) and soft-bottom stations (mean $\pm \mathrm{SD}$ : $1.78 \pm 0.50)$, and did not differ significantly between water masses $(p=0.475$, ANOVA) or clusters ( $\mathrm{p}=0.468$, ANOVA). Similarly, Pielou's $J^{\prime}$ was not different between hard-bottom (mean \pm SD: $0.56 \pm 0.16$ ) and soft-bottom stations $($ mean $\pm \mathrm{SD}: 0.58 \pm 0.17)$, clusters $(\mathrm{p}=$ 0.390, ANOVA), or water masses ( $\mathrm{p}=$ 0.266 , ANOVA). $J^{\prime}$ and $H^{\prime}$ were negatively correlated with total biomass $\left(J^{\prime}\right.$ : Pearson correlation coefficient $=-0.632$, $\mathrm{p}<0.001$, Bartlett's chi-square $=12.476$; $H$ : Pearson correlation coefficient $=$ $-0.499, \mathrm{p}<0.008$, Bartlett's chi-square $=7.018, \mathrm{n}=27$ for both). Once mobile and sessile biomass were separated, however, there was no correlation of diversity indices $J^{\prime}$ and $H^{\prime}$ with sessile biomass. A Pearson correlation of epifaunal and macrofaunal biomass failed to show a significant correlation (Pearson coefficient = 0.073, $\mathrm{p}=0.575$, Bartlett's chi-square $=0.315, \mathrm{n}=61$; Fig. 8). 


\section{Group average}

a

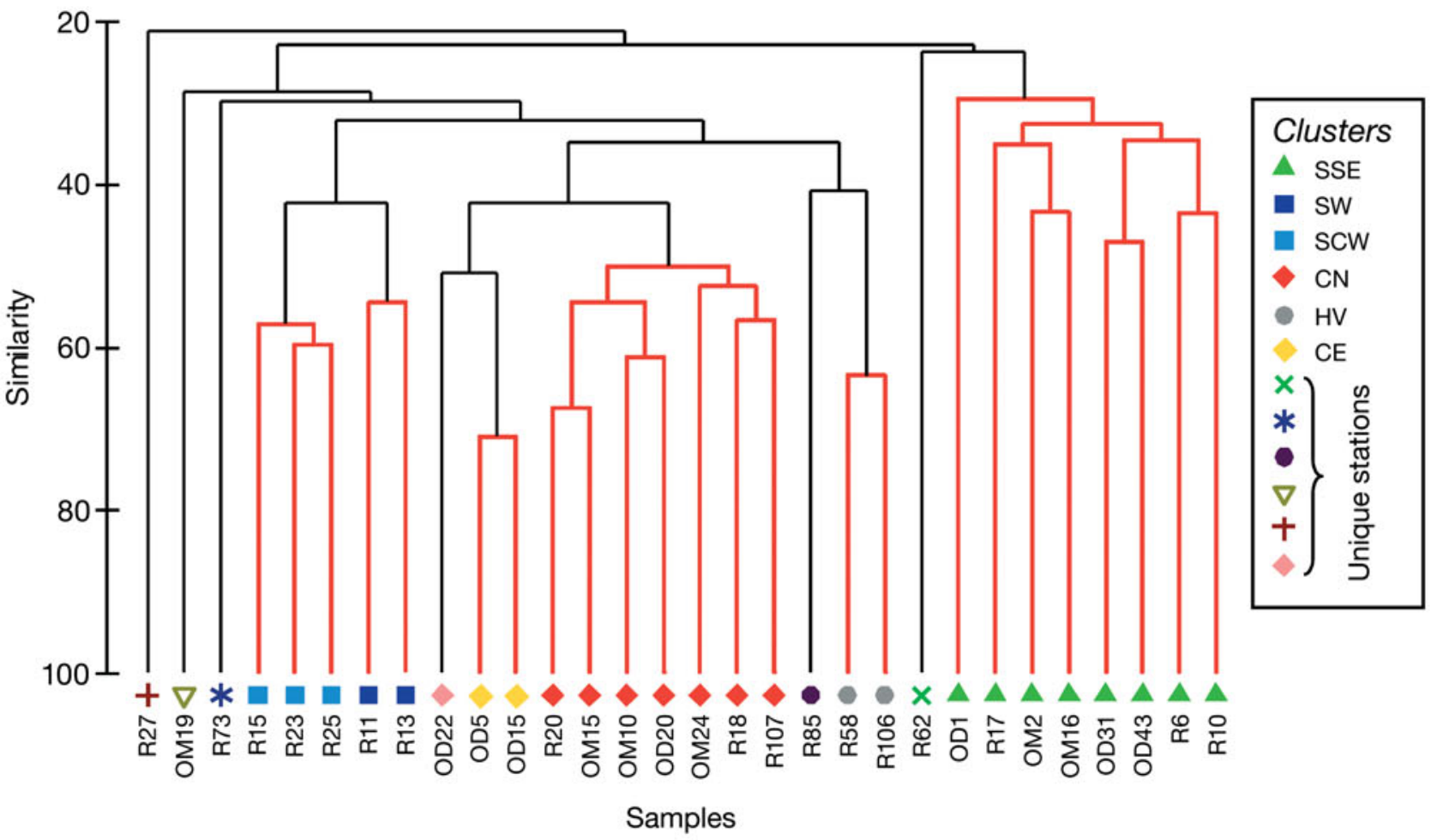

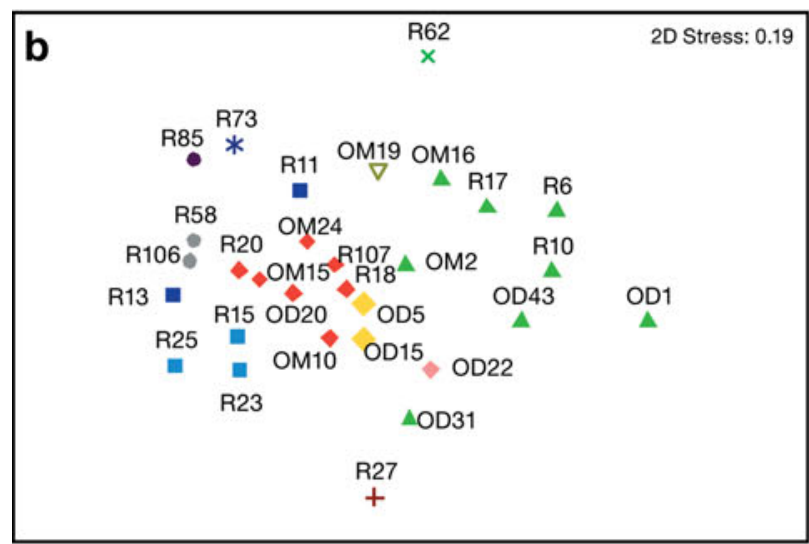

Fig. 6. Station similarity. Color code is shared in all panels. (a) Station clusters obtained from hierarchical clustering of the epifaunal biomass matrix (fourth-root-transformed, BrayCurtis similarity). Red lines connect stations that are not statistically unique ( $p=0.05$, SIMPROF). (b) NMDS of the epifaunal biomass matrix. (c) Spatial distribution of epifaunal clusters in the Chukchi Sea, with unique stations marked with black. Note: 2008 data were excluded (see 'Materials and methods'). For abbreviations of cluster designations see Table 2

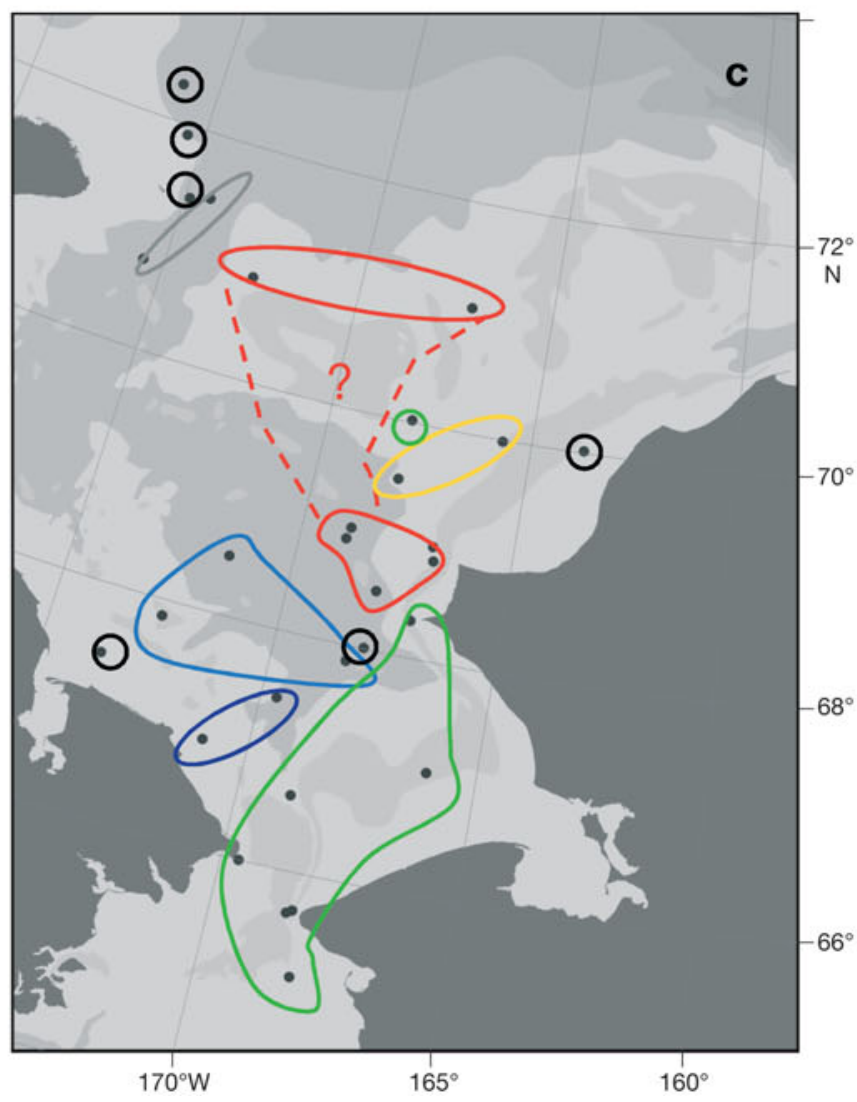


Table 3. Top 3 epifaunal species contributing most to dissimilarity between each of the 6 main clusters identified from hierarchical clustering. for other abbreviations see Table 2

\begin{tabular}{|c|c|c|c|c|c|c|}
\hline \multirow{2}{*}{$\begin{array}{l}\text { Species } \\
\text { Groups SSE \& SW, average dissimilarity }=\mathbf{7 9} \%\end{array}$} & \multicolumn{2}{|c|}{$\begin{array}{l}\text { Average biomass } \\
\text { (g wet wt. } 100 \mathrm{~m}^{-2} \text { ) }\end{array}$} & \multirow[t]{2}{*}{$\begin{array}{c}\text { Average } \\
\text { dissimilarity }\end{array}$} & \multirow[t]{2}{*}{$\begin{array}{c}\text { SD of } \\
\text { dissimilarity }\end{array}$} & \multirow[t]{2}{*}{$\begin{array}{l}\text { Contrib. } \\
(\%)\end{array}$} & \multirow[t]{2}{*}{$\underset{(\%)}{\mathrm{Cum}}$} \\
\hline & SSE & SW & & & & \\
\hline Myriotrochus rinkii & 0.0 & 9.9 & 5.0 & 5.0 & 6.3 & 6.3 \\
\hline Strongylocentrotus pallidus & 6.9 & 0.0 & 3.4 & 1.4 & 4.3 & 10.6 \\
\hline Cryptonatica affinis & 0.5 & 5.9 & 2.8 & 2.1 & 3.5 & 14.0 \\
\hline Groups SSE \& SCW, average dissimilarity $=80 \%$ & SSE & SCW & & & & \\
\hline Strongylocentrotus pallidus & 6.9 & 0.0 & 3.6 & 1.4 & 4.5 & 4.5 \\
\hline Cryptonatica affinis & 0.5 & 5.5 & 2.7 & 2.6 & 3.3 & 7.9 \\
\hline Amphiodia craterodmeta & 0.3 & 5.0 & 2.6 & 1.7 & 3.2 & 11.1 \\
\hline Groups SW \& SCW, average dissimilarity $=88 \%$ & SW & SCW & & & & \\
\hline Myriotrochus rinkii & 9.9 & 4.4 & 3.3 & 4.5 & 5.7 & 5.7 \\
\hline Cribrinopsis/Epiactis sp. & 6.6 & 1.2 & 3.3 & 1.7 & 5.6 & 11.3 \\
\hline Urticina sp.? & 0.0 & 5.5 & 3.2 & 6.1 & 5.6 & 16.9 \\
\hline Groups SSE \& CN, average dissimilarity $=74 \%$ & SSE & $\mathbf{C N}$ & & & & \\
\hline Strongylocentrotus pallidus & 6.9 & 0.0 & 3.6 & 1.4 & 4.9 & 4.9 \\
\hline Ophiura sarsi & 0.5 & 6.0 & 3.1 & 1.0 & 4.2 & 9.0 \\
\hline Buccinum elatior & 0.0 & 4.5 & 2.4 & 3.3 & 3.2 & 12.3 \\
\hline Groups SW \& CN, average dissimilarity $=69 \%$ & SW & $\mathbf{C N}$ & & & & \\
\hline Myriotrochus rinkii & 9.9 & 0.0 & 5.8 & 12.1 & 8.5 & 8.5 \\
\hline Cribrinopsis/Epiactis sp. & 6.6 & 0.0 & 3.9 & 2.6 & 5.7 & 14.2 \\
\hline Henricia tumida & 6.3 & 0.0 & 3.7 & 12.1 & 5.4 & 19.6 \\
\hline Groups SCW \& CN, average dissimilarity $=65 \%$ & SCW & $\mathbf{C N}$ & & & & \\
\hline Urticina sp.? & 5.5 & 0.0 & 3.5 & 5.7 & 5.3 & 5.3 \\
\hline Ophiura sarsi & 2.4 & 6.0 & 3.4 & 1.1 & 5.1 & 10.4 \\
\hline Amphiodia craterodmeta & 5.0 & 0.0 & 3.2 & 2.0 & 4.9 & 15.3 \\
\hline Groups SSE \& HC, average dissimilarity $=83 \%$ & SSE & HC & & & & \\
\hline Ctenodiscus crispatus & 0.0 & 10.8 & 5.7 & 4.6 & 6.9 & 6.9 \\
\hline Strongylocentrotus pallidus & 6.9 & 0.0 & 3.6 & 1.4 & 4.3 & 11.2 \\
\hline Amphiodia craterodmeta & 0.3 & 6.4 & 3.4 & 1.0 & 4.0 & 15.2 \\
\hline Groups SW \& HC, average dissimilarity $=63 \%$ & SW & HC & & & & \\
\hline Ctenodiscus crispatus & 0.0 & 10.8 & 6.3 & 21.7 & 10.1 & 10.1 \\
\hline Myriotrochus rinkii & 9.9 & 0.8 & 5.4 & 8.1 & 8.5 & 18.6 \\
\hline Cribrinopsis/Epiactis sp. & 6.6 & 0.0 & 3.9 & 2.4 & 6.2 & 24.8 \\
\hline Groups SCW \& HC, average dissimilarity $=63 \%$ & SCW & HC & & & & \\
\hline Ctenodiscus crispatus & 0.0 & 10.8 & 6.8 & 13.1 & 10.8 & 10.8 \\
\hline Amphiodia craterodmeta & 5.0 & 6.4 & 4.1 & 2.3 & 6.4 & 17.2 \\
\hline Tachyrhynchus erosus & 0.0 & 5.9 & 3.8 & 6.3 & 5.9 & 23.1 \\
\hline Groups $\mathrm{CN} \& \mathrm{HC}$, average dissimilarity $=59 \%$ & $\mathbf{C N}$ & $\mathrm{HC}$ & & & & \\
\hline Ctenodiscus crispatus & 0.0 & 10.8 & 6.8 & 10.4 & 11.6 & 11.6 \\
\hline Amphiodia craterodmeta & 0.0 & 6.4 & 4.0 & 1.0 & 6.8 & 18.4 \\
\hline Ophiura sarsi & 6.0 & 0.0 & 3.7 & 1.0 & 6.3 & 24.7 \\
\hline Groups SSE \& CE, average dissimilarity $=72 \%$ & SSE & $\mathbf{C E}$ & & & & \\
\hline Strongylocentrotus pallidus & 6.9 & 0.0 & 4.2 & 1.4 & 5.9 & 5.9 \\
\hline Leptasterias groenlandica & 5.0 & 0.0 & 2.9 & 2.7 & 4.0 & 9.9 \\
\hline Leptasterias polaris & 5.3 & 4.7 & 2.9 & 1.5 & 4.0 & 14.0 \\
\hline Groups SW \& CE, average dissimilarity $=70 \%$ & SW & CE & & & & \\
\hline Myriotrochus rinkii & 9.9 & 0.0 & 6.9 & 48.8 & 9.8 & 9.8 \\
\hline Cribrinopsis/Epiactis sp. & 6.6 & 0.0 & 4.6 & 2.4 & 6.6 & 16.4 \\
\hline Gorgonocephalus caryi & 0.0 & 6.1 & 4.3 & 4.4 & 6.1 & 22.5 \\
\hline Groups SCW \& CE, average dissimilarity $=69 \%$ & SCW & $\mathrm{CE}$ & & & & \\
\hline Gorgonocephalus caryi & 0.0 & 6.1 & 4.7 & 4.4 & 6.7 & 6.7 \\
\hline Urticina sp.? & 5.5 & 0.0 & 4.2 & 6.2 & 6.0 & 12.8 \\
\hline Amphiodia craterodmeta & 5.0 & 0.0 & 3.9 & 1.9 & 5.6 & 18.4 \\
\hline Groups CN \& CE, average dissimilarity $=54 \%$ & $\mathbf{C N}$ & $\mathrm{CE}$ & & & & \\
\hline Gorgonocephalus caryi & 0.0 & 6.1 & 4.7 & 4.3 & 8.7 & 8.7 \\
\hline Ophiura sarsi & 6.0 & 0.9 & 4.1 & 1.0 & 7.6 & 16.3 \\
\hline Neptunea communis & 3.3 & 0.0 & 2.4 & 2.2 & 4.5 & 20.8 \\
\hline Groups HC \& CE, average dissimilarity $=67 \%$ & HC & $\mathbf{C E}$ & & & & \\
\hline Ctenodiscus crispatus & 10.8 & 0.0 & 8.2 & 23.3 & 12.2 & 12.2 \\
\hline Amphiodia craterodmeta & 6.4 & 0.0 & 4.8 & 0.9 & 7.1 & 19.3 \\
\hline Gorgonocephalus caryi & 0.0 & 6.1 & 4.7 & 4.4 & 6.9 & 26.2 \\
\hline
\end{tabular}


Table 4. The 5 dominant epibenthic taxa in each of the 6 main clusters identified from cluster analysis, their contribution to total cluster biomass and their mean biomass $( \pm \mathrm{SD})$ at the cluster stations (see Fig. 6 for station clusters). na: not applicable

\begin{tabular}{|c|c|c|c|c|}
\hline Cluster & Dominant taxa by biomass & $\begin{array}{l}\text { Percent of total } \\
\text { cluster biomass }\end{array}$ & $\begin{array}{c}\text { Mean biomass } \\
\text { (g wet wt } 1000 \mathrm{~m}^{-2} \text { ) }\end{array}$ & $\mathrm{SD}$ \\
\hline South/southeast (SSE) & $\begin{array}{l}\text { Strongylocentrotus pallidus } \\
\text { Leptasterias polaris } \\
\text { Chionoecetes opilio } \\
\text { Lethasterias nanimensis } \\
\text { Hyas coarctatus } \\
\text { Sum/mean total biomass }\end{array}$ & $\begin{array}{r}24.6 \\
14.2 \\
8.4 \\
5.1 \\
4.9 \\
57.3\end{array}$ & $\begin{array}{r}8942 \\
5172 \\
3071 \\
1867 \\
1778 \\
34455\end{array}$ & $\begin{array}{r}12013 \\
7122 \\
6526 \\
4420 \\
2005 \\
26536\end{array}$ \\
\hline Southwest (SW) & $\begin{array}{l}\text { Myriotrochus rinkii } \\
\text { Chionoecetes opilio } \\
\text { Cribrinopsis sp. } \\
\text { Cryptonatica affinis } \\
\text { Henricia tumida } \\
\text { Sum/mean total biomass }\end{array}$ & $\begin{array}{r}40.7 \\
15.8 \\
14.1 \\
8.3 \\
6.7 \\
85.7\end{array}$ & $\begin{array}{r}9602 \\
3735 \\
3318 \\
1964 \\
1578 \\
23579\end{array}$ & $\begin{array}{r}179 \\
4489 \\
4240 \\
2387 \\
45 \\
9757\end{array}$ \\
\hline South-central-west (SCW) & $\begin{array}{l}\text { Amphiodia craderodmeta } \\
\text { Cryptonatica affinis } \\
\text { Urticina sp. } \\
\text { Chionoecetes opilio } \\
\text { Myriotrochus rinkii } \\
\text { Sum/mean total biomass }\end{array}$ & $\begin{array}{r}14.5 \\
13.8 \\
12.5 \\
9.9 \\
5.7 \\
56.4\end{array}$ & $\begin{array}{r}1241 \\
1184 \\
1072 \\
851 \\
493 \\
8590\end{array}$ & $\begin{array}{r}1093 \\
869 \\
901 \\
649 \\
469 \\
979\end{array}$ \\
\hline Central \& north $(\mathrm{CN})$ & $\begin{array}{l}\text { Chionoecetes opilio } \\
\text { Ophiura sarsi } \\
\text { Pagurus rathbuni } \\
\text { Leptasterias polaris } \\
\text { Neptunea ventricosa } \\
\text { Sum / mean total biomass }\end{array}$ & $\begin{array}{r}39.7 \\
25.1 \\
13.0 \\
3.9 \\
2.7 \\
84.4\end{array}$ & $\begin{array}{r}8870 \\
5603 \\
2896 \\
866 \\
598 \\
22326\end{array}$ & $\begin{array}{r}16072 \\
10951 \\
4204 \\
722 \\
662 \\
21843\end{array}$ \\
\hline Herald Canyon (HC) & $\begin{array}{l}\text { Ctenodiscus crispatus } \\
\text { Amphiodia craderodmeta } \\
\text { Chionoecetes opilio } \\
\text { Urasterias linki } \\
\text { Tachyrhynchus erosus } \\
\text { Sum/mean total biomass }\end{array}$ & $\begin{array}{r}34.5 \\
34.1 \\
11.4 \\
9.8 \\
3.3 \\
93.0\end{array}$ & $\begin{array}{r}13715 \\
13554 \\
4553 \\
3881 \\
1317 \\
39788\end{array}$ & $\begin{array}{r}4345 \\
\text { na } \\
1087 \\
\text { na } \\
793 \\
16023\end{array}$ \\
\hline Central-east (CE) & $\begin{array}{l}\text { Gorgonocephalus caryi } \\
\text { Leptasterias polaris } \\
\text { Chionoecetes opilio } \\
\text { Neptunea ventricosa } \\
\text { Pagurus rathbuni } \\
\text { Sum/mean total biomass }\end{array}$ & $\begin{array}{r}34.1 \\
21.1 \\
15.8 \\
6.4 \\
5.3 \\
82.6\end{array}$ & $\begin{array}{r}1643 \\
1013 \\
759 \\
306 \\
255 \\
4810\end{array}$ & $\begin{array}{r}1445 \\
1352 \\
586 \\
190 \\
265 \\
1047\end{array}$ \\
\hline
\end{tabular}

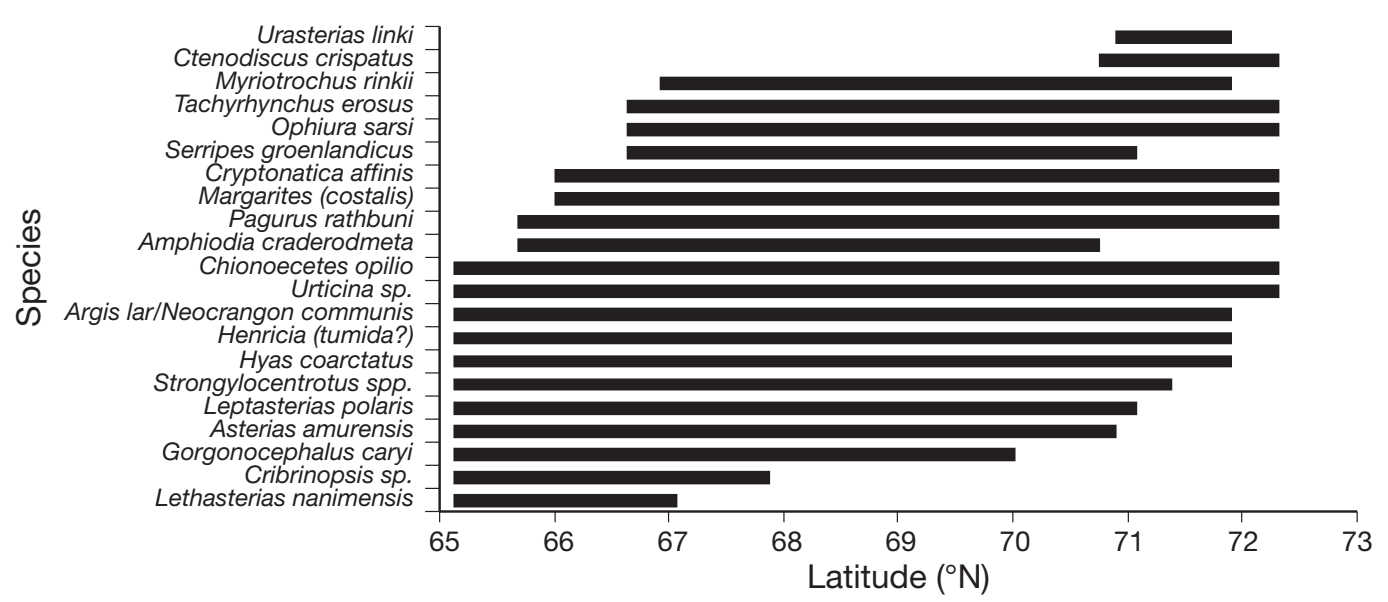

Fig. 7. Latitudinal ranges of dominant species found in the study area. Dominant taxa include the top 5 contributors to abundance and biomass in each of the cluster groups (see Table 2, Fig. 6), as well as any additional taxa that dominated at a single station and occurred at $>3$ stations 
Table 5. Results of the BIO-ENV procedure providing the combinations of environmental variables best matching the community similarity matrix, based on biomass. Correlation coefficients are given in parentheses. In addition to the variables given below, macrofaunal biomass and integrated chlorophyll concentration were included in the analysis, but did not strongly influence biotic variables

\begin{tabular}{|lll|}
\hline $\begin{array}{l}\text { Number of } \\
\text { variables }\end{array}$ & Best variable combination & Second best variable combination \\
\hline 1 & Latitude $(0.379)$ & \\
2 & Latitude, substrate $(0.503)$ & Substrate $(0.363)$ \\
3 & Latitude, substrate, salinity (0.535) & Latitude, salinity (0.442) \\
4 & Latitude, substrate, salinity, organic content (0.531) & Water depth, latitude, substrate (0.510) \\
5 & Latitude, substrate, grain size, temperature, & Water depth, latitude, substrate, salinity (0.526) \\
& organic content $(0.542)$ & Latitude, substrate, grain size, salinity, \\
6 & Water depth, latitude, substrate, grain size, & Water depth, latitude, substrate, grain size, salinity, \\
& Salinity, oxygen (0.549) & organic content (0.547) \\
7 & Water depth, latitude, substrate, grain size, & Water depth, latitude, substrate, grain size, salinity, \\
& temperature, oxygen, organic content $(0.553)$ & oxygen, organic content $(0.552)$ \\
\hline
\end{tabular}

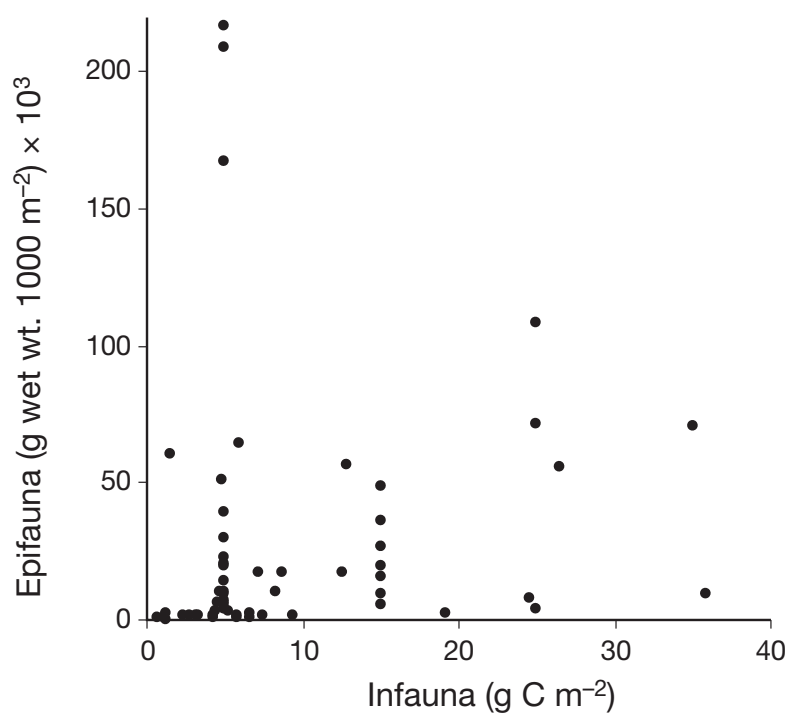

Fig. 8. Relationship betwee macrofauna and epifauna, no significant correlation (Pearson coefficient $=0.073, \mathrm{p}=0.575$, Bartlett's chi-square $=0.315, \mathrm{n}=61$ )

\section{DISCUSSION}

\section{Community composition}

Echinoderms and crustaceans dominated epifaunal abundance and biomass on the Chukchi shelf, while gastropods were the most species-rich taxon (Figs. 2b, $3 \mathrm{~b} \& 5 \mathrm{~b})$. The dominance of echinoderms is consistent with previous studies of both shelf and deep-sea regions of the Arctic (see references in Table 6 and MacDonald et al. in press). All echinoderm classes, except crinoids, were commonly encountered on the Chukchi shelf, with ophiuroids most prevalent in the northern areas and asteroids dominating in the southern Chukchi and the northern Bering Seas (Table 6). Certain sea stars, such as Ctenodiscus crispatus, only occurred at the northern stations, but did not significantly influence total biomass.

The single most abundant brittle star (and echinoderm) we encountered in the Chukchi Sea was Ophiura sarsi (see also Frost \& Lowry 1983, Ambrose et al. 2001, Feder et al. 2005, Sirenko et al. unpubl. data), a wideranging boreal-Arctic species with circumpolar distribution (Anisimova 1989). O. sarsi is also common in the adjacent Beaufort Sea (Carey 1977, Loggerwell \& Rand 2009) and in the Japanese Pacific (Fujita \& Ohta 1989). On Eurasian Arctic shelves, the much smaller brittle star Ophiocten sericeum dominates (Starmans et al. 1999, Piepenburg 2000; see also Table 7), although O. sarsi is also common in Svalbard waters (Piepenburg et al. 1996). Like $O$. sarsi, O. sericeum is also described as a wide-ranging boreal-Arctic species (Anisimova 1989), but was rarely encountered in our Chukchi Sea samples.

The abundance of Ophiura sarsi was of the same order of magnitude as previously reported from an inshore region in the northeastern Chukchi Sea (Ambrose et al. 2001), but higher than in the southeastern Chukchi/Kotzebue Sound area (Feder et al. 2005) (Table 7). The cause for a north to south decrease in ophiuroid dominance is unclear. Worldwide, dense ophiuroid beds have been discovered in most marine habitats, including continental shelves, slopes and abyssal plains (Table 7), and are thought to favor areas where predation pressure is low (Aronson \& Sues 1987). For example, O. sarsi is reported to exhibit avoidance behavior in the presence of mobile sea stars and buccinid snails (Fujita \& Ohta 1989), although we found Buccinidae to be very abundant in both the 
Table 6. Dominant epibenthic taxa by biomass in the northern Bering, Chukchi and Beaufort Seas. Note that Strongylocentrotus droebachiensis in Feder et al. (2005) is probably in fact $S$. pallidus (H. M. Feder pers. comm.). Neptunea heros in Feder et al. (2005) is referred to as N. ventricosa in the Russian and European literature and in the present study

\begin{tabular}{|c|c|c|}
\hline Study area & Dominant taxa by biomass & Source \\
\hline Chukchi Sea & $\begin{array}{l}\text { Ophiura sarsi, Chionoecetes opilio, Strongylocentrotus pallidus, } \\
\text { Leptasterias polaris }^{\mathrm{a}}\end{array}$ & Present study \\
\hline Northeastern Chukchi Sea & $\begin{array}{l}\text { Ophiuroids (Ophiura. sarsi, O. maculata, Ophiopholis aculeata, } \\
\text { Stegophiura nodosa), Echinarachnius parma }\end{array}$ & Ambrose et al. 2001 \\
\hline $\begin{array}{l}\text { Southern Chukchi Sea, } \\
\text { Kotzebue Sound }\end{array}$ & $\begin{array}{l}\text { Asterias amurensis, Neptunea heros, Leptasterias polaris, } \\
\text { Chionoecetes opilio, Strongylocentrotus droebachiensis, } \\
\text { Lethasterias nanimensis }{ }^{\mathrm{a}}\end{array}$ & Feder et al. 2005 \\
\hline Norton Sound & $\begin{array}{l}\text { Sea stars (Asterias amurensis, Lethasterias nanimensis, } \\
\text { Evasterias spp. })^{\mathrm{b}}\end{array}$ & Hamazaki et al. 2005 \\
\hline $\begin{array}{l}\text { Northeastern Bering Sea } \\
\text { (Chirikov Basin and } \\
\text { Norton Sound) }\end{array}$ & $\begin{array}{l}\text { Asterias amurensis, Gorgonocephalus caryi, } \\
\text { Lethasterias nanimensis }\end{array}$ & Jewett \& Feder 1981 \\
\hline Southeastern Bering Sea & $\begin{array}{l}\text { Chionoecetes opilio, C. bairdi, Paralithodes camtschatika, } \\
\text { Asterias amurensis, Styela rustica }{ }^{\mathrm{a}}\end{array}$ & Jewett \& Feder 1981 \\
\hline Alaskan Beaufort Sea & Ophiura sarsi, Chionoecetes opilio, Musculus niger ${ }^{\mathrm{a}}$ & Loggerwell \& Rand 2009 \\
\hline
\end{tabular}

Table 7. Ophiuroid abundance and biomass in the Arctic and comparative areas

\begin{tabular}{|c|c|c|c|c|}
\hline Species & $\begin{array}{c}\text { Density range } \\
\text { (average all stations) } \\
\text { (ind. } \mathrm{m}^{-2} \text { ) }\end{array}$ & $\begin{array}{c}\text { Biomass range } \\
\text { (average all stations) } \\
\text { (g AFDM) }\end{array}$ & Study area & Source (and method) \\
\hline Ophiura sarsi & $0-99.9$ & $0-3.8$ & NE Chukchi Sea & $\begin{array}{l}\text { Ambrose et al. } 2001 \\
\text { (video plus dredge) }\end{array}$ \\
\hline Ophiura sarsi & $\begin{array}{c}\text { 0-0.1 (reported as } \\
0-108267 \text { ind. } \mathrm{km}^{-2} \text { ) }\end{array}$ & & $\begin{array}{l}\text { Kotzebue Sound, } \\
\text { SE Chukchi Sea }\end{array}$ & $\begin{array}{l}\text { Feder et al. } 2005 \\
\text { (otter trawl) }\end{array}$ \\
\hline Ophiura sarsi & $\begin{array}{c}0-68.1 \\
(5.5)\end{array}$ & $\begin{array}{l}0-3.7^{\mathrm{a}} \\
\left(0.3^{\mathrm{a}}\right.\end{array}$ & Chukchi Sea & Present study (beam trawl) \\
\hline Ophiura sarsi & $\begin{array}{c}353 \\
\text { (1 station only) }\end{array}$ & $8.4^{\mathrm{a}}$ & $\begin{array}{l}\text { North Pacific } \\
\text { off Japan }\end{array}$ & $\begin{array}{l}\text { Fujita \& Ohta } 1989 \\
\text { (still images plus trawls) }\end{array}$ \\
\hline $\begin{array}{l}\text { Ophiocten sericeum } \\
\text { Ophiura sarsi }\end{array}$ & $\begin{array}{r}0.3-566(158) \\
0.4-35(5.6)\end{array}$ & $\begin{array}{l}<0.1-1.5(0.5) \\
<0.1-3.8(0.5)\end{array}$ & Laptev Sea & $\begin{array}{l}\text { Piepenburg \& Schmid } 1997 \\
\text { (still images plus dredge) }\end{array}$ \\
\hline $\begin{array}{l}\text { Ophiocten sericeum, } \\
\text { Ophiacantha bidentata }\end{array}$ & $\begin{array}{c}32-524 \\
2-49\end{array}$ & $\begin{array}{l}0.3-5.0 \\
0.1-1.9\end{array}$ & Barents Sea & $\begin{array}{l}\text { Piepenburg \& Schmid } 1996 \\
\text { (still images \& trawl) }\end{array}$ \\
\hline Ophiocten sericeum & (6) & & $\begin{array}{l}\text { NE Greenland } \\
\left(79-81.3^{\circ} \mathrm{N}\right)\end{array}$ & Starmans et al. 1999 (video) \\
\hline Ophiocten sericeum & $(5.3)$ & & $\begin{array}{l}\text { NE Greenland } \\
\left(75^{\circ} \mathrm{N}\right)\end{array}$ & $\begin{array}{l}\text { Mayer \& Piepenburg } 1996 \\
\text { (still images) }\end{array}$ \\
\hline Ophiacantha abyssicola & $390-1200$ & & $\begin{array}{l}\text { Continental slope } \\
\text { of Nova Scotia }\end{array}$ & $\begin{array}{l}\text { Metaxas \& Griffin } 2004 \\
\text { (still images) }\end{array}$ \\
\hline
\end{tabular}

southern and the northern Chukchi Sea (Feder et al. 1994a, 2005, present study). Further, sea stars in Norton Sound and the southeastern Chukchi Sea are not known to feed on ophiuroids (Feder \& Jewett 1978). Flatfishes and Chionoecetes opilio in the southeastern Bering Sea, however, do utilize ophiuroids as a food source (Feder \& Jewett 1980, 1981), and both taxa are less prevalent in the northern than in the southern
Chukchi Sea (Barber et al. 1997, Mecklenburg et al. 2007, present study), possibly contributing to the dominance of ophiuroids in the north.

Dense patches of the gelatinous apodid holothurian Myriotrochus rinkii occurred at some soft-bottom stations where Ophiura sarsi was uncommon or absent. M. rinkii is also common in other Arctic shelf areas such as the Laptev Sea and around Baffin Island (Cross 
\& Thomson 1987, Piepenburg \& Schmid 1997). Biomass in this taxon was particularly high in areas with high nutrient and chlorophyll concentrations (Lee et al. 2007). Holothurians are known to form these dense assemblages to exploit fresh phytodetritus pulses on soft bottoms. Examples include Amperima rosea aggregations in the Porcupine Abyssal Plain in the North Atlantic (Billett et al. 2001, Iken et al. 2001), high densities of Kolga hyalina in a phytodetritus-rich, pockmark-like depression on the Chukchi Cap in the Arctic Ocean (MacDonald et al. in press), and high densities of Cucumaria frondosa at the bottom of pockmarks in the Bay of Fundy (Wildish et al. 2008). This type of opportunistic feeding behavior suggests that holothurians may play a significant role in remineralization of newly deposited organic material, and could characterize regions of tight benthic-pelagic coupling on Arctic shelves.

Crustaceans were the second most abundant and biomass-rich group in the Chukchi Sea, particularly at eastern stations (Figs. 2b, 3b \& 4). This pattern contrasts with the trend suggested by Feder et al. (2005) of epifaunal crustacean dominance in the south and echinoderm dominance in the north. However, over a larger spatial scale that includes the Bering Sea, the trend may indeed hold true (Table 6) and has been attributed to the smaller populations of large crabs and demersal fishes in the north (Barber et al. 1997, Paul et al. 1997, Feder et al. 2005), which provide vacant niches for other taxa. Alternatively, physiological differences may allow long-lived, slow-growing taxa such as echinoderms to dominate in very cold and food-limited parts of polar waters (Dahm 1996, Bluhm et al. 1998), while distributions of other taxa, such as crabs, are constrained at the sub-zero temperatures typical of the northern Chukchi (Frederich et al. 2000, present study).

Despite physiological constraints, crabs did constitute the most biomass-rich epifaunal crustacean group in the Chukchi Sea, dominated mainly by Chionoecetes opilio, but also with high local densities of several species of hermit crabs and the lyre crab Hyas coarctatus in some areas (see also Feder et al. 2005). In contrast, crabs are typically uncommon on other Arctic shelves (Mayer \& Piepenburg 1996, Piepenburg et al. 1996, Starmans et al. 1999). The conspicuous scarcity of crabs in Antarctic waters (Thatje \& Arntz 2004) has been attributed to the narcotizing effect of high magnesium concentrations in the haemolymph of some organisms at low temperatures, including Chionoecetes spp. (Frederich et al. 2000). However, C. opilio is metabolically restricted to water temperatures $<5^{\circ} \mathrm{C}$ and is commonly found at temperatures between 0 and $1^{\circ} \mathrm{C}$. It also occurs at sub-zero temperatures, even though it exhibits negative growth below $1^{\circ} \mathrm{C}$ (Taylor \& O'Keefe 1986, Foyle et al. 1989). The records of $C$. opilio reported here, to our knowledge, represent an expansion of the northernmost range limit of the species in the Pacific Arctic into Herald Canyon $\left(72.30^{\circ} \mathrm{N}\right.$; Fig. 4), where water temperatures were below zero, although $C$. opilio biomass was low at these sites. In the eastern Atlantic, a recently established $C$. opilio population now occurs as far north as $76^{\circ} \mathrm{N}$ in water temperatures from -0.7 to $3.4^{\circ} \mathrm{C}$, with most crabs found in waters $<2^{\circ} \mathrm{C}$ (Alvsvåg et al. 2009).

While echinoderms and crustaceans were the dominant contributors to biomass and abundance, gastropods were the most species-rich group at our sites (Fig. 5b), which supports findings from earlier studies (Frost \& Lowry 1983, Feder et al. 2005). Dominant taxa by biomass included the families Buccinidae and Naticidae (moon snails). Often preying on bivalves and polychaetes (Morton \& Jones 2003), these carnivores and opportunistic scavengers find abundant food in the Chukchi Sea (Sirenko \& Gagaev 2007). Solariella obscura and S. varicosa (Trochidae), grazers feeding on algae and small detritus, were particularly abundant in the south-central Chukchi Sea. High water-column chlorophyll concentrations (Lee et al. 2007) coupled with abundant epifaunal grazers and high macrofaunal biomass (Grebmeier et al. 2006a, Sirenko \& Gagaev 2007) suggest that the transfer of detritus from the sediment into the benthic food web may be particularly efficient in this region.

\section{Possible temporal trends in Chukchi Sea epifauna}

While speculations regarding temporal trends in Chukchi Sea epifaunal communities are tenuous, particularly due to the limited number of stations sampled repetitively and the inconsistencies in sampling gear types, we offer here a cautious discussion of observed patterns in an effort to help direct future work. Only one of our stations overlapped with a station sampled by Ambrose et al. (2001) with an ROV in 1998; at their Stn 4, the sand dollar Echinarachnius parma dominated with abundances of 124 ind. $\mathrm{m}^{-2}$. In 1986, near the stations Ambrose et al. (2001) sampled, Feder et al. (1994b) found E. parma in a mean abundance of 276 ind. $\mathrm{m}^{-2}$ (their group IV). In 2007, the sand dollar was still dominant in this area, although abundance estimates (Stn OM19) were much lower (4 ind. $\mathrm{m}^{-2}$ ), suggesting a potential decrease of this taxon in the area. Earlier reports mention a band of sand dollars under the frontal zone between BSW and ACW just north of the Bering Strait (Grebmeier et al. 1989), where we did not find the species.

Preliminary comparisons of biomass and community composition at 8 station pairs in the southeastern Chukchi Sea sampled in both the present study (2004 
to 2008) and by other researchers in 1976 (Feder et al. 2005) indicated that 1976 biomass estimates ranged from 1 to $67 \%$ of present-day estimates. Chionoecetes opilio had increased in relative total abundance at 6 stations, and in biomass at all 8 stations (Table 8). Absolute crab biomass was 1 to 2 orders of magnitude higher in the present study than in the 1976 study, and was dominated in terms of biomass at 5 of the 8 stations in the present study, compared to none in 1976. Sparks \& Pereyra (1966) did not mention C. opilio as a dominant taxon in their 1959 trawl collections either, but listed the species as occurring, as did MacGinitie (1955) with reference to his collection of C. opilio at several stations in from 10 to $145 \mathrm{~m}$ depth off Barrow, Alaska. A trend toward increased biomass in the 2000s, in particular for C. opilio, agrees with Feder et al.'s (2005) comparison of their own data in 1976 to a fisheries survey in 1998 (Fair \& Nelson 1999), in which biomass had at least doubled for several dominant groups in this period of time and average C. opilio abundance had increased by an order of magnitude. On a pan-Arctic scale, $C$. opilio concentrations have shifted northward in the Bering Sea (Orensanz et al. 2004, http://access.afsc.noaa.gov/reem/ecoweb/html/ EcoContribution.cfm? ID=38), and, in the European Arctic, a self-sustained population now occurs in the Barents Sea (Alvsvåg et al. 2008), where the first $C$. opilio were documented in 1996 (Kuzmin et al. 1998). These shifts are not only potential ecological indicators of climate warming, but also have clear implications for the current sub-Arctic crab fishery, as well as for any future Arctic fisheries that may develop. Based on the temperature preferences of $C$. opilio (see above), further northward range extensions or changes in biomass centers might only occur if average temperatures increase in those areas.

Table 8. Comparison of dominant species, with a focus on Chionoecetes opilio, by biomass at 8 locations in the southern Chukchi Sea in Feder et al. (2005) and in the present study. Note that Strongylocentrotus droebachiensis in Feder et al. (2005) is probably in fact $S$. pallidus (H. M. Feder pers. comm.). Neptunea heros in Feder et al. (2005) is referred to as N. ventricosa in the Russian and European literature and in the present study. $N$. ventricosa in Feder et al. (2005) is referred to as $N$. behringiana in the European and Russian literature. Species among the 5 dominant taxa at a given station in both studies are given in bold print. Species are underlined if they were common at many of the compared stations in Feder et al. (2005), but never or rarely observed in the present study

\begin{tabular}{|c|c|c|c|c|c|c|c|}
\hline \multirow{2}{*}{\multicolumn{2}{|c|}{ Station number }} & \multirow{2}{*}{\multicolumn{4}{|c|}{ Biomass (g wet wt $1000 \mathrm{~m}^{-2}$ ) }} & \multirow{2}{*}{\multicolumn{2}{|c|}{ - Dominant species by biomass }} \\
\hline & & & & & & & \\
\hline $\begin{array}{l}\text { Present } \\
\text { study }\end{array}$ & $\begin{array}{l}\text { Feder } \\
\text { et al. }\end{array}$ & $\begin{array}{l}\text { Present } \\
\text { study }\end{array}$ & $\begin{array}{l}\text { Feder } \\
\text { et al. }\end{array}$ & $\begin{array}{l}\text { Present } \\
\text { study }\end{array}$ & $\begin{array}{l}\text { Feder } \\
\text { et al. }\end{array}$ & Present study & Feder et al. \\
\hline R6 & A54 & 68630 & 6106 & 1564 & 36 & $\begin{array}{l}\text { Strongylocentrotus pallidus, } \\
\text { Leptasterias groenlandicus, } \\
\text { Stomphia sp., Gersemia sp., } \\
\text { Hyas coarctatus }\end{array}$ & $\begin{array}{l}\text { S. droebachiensis, } \\
\text { Psolus japonicus, } \\
\text { H. coarctatus, } \\
\text { Lethasterias nanimensis, } \\
\text { Paralithodes platypus }\end{array}$ \\
\hline OM2 & A36 & 71864 & 746 & 19103 & 90 & $\begin{array}{l}\text { Chionoecetes opilio, } \\
\text { Leptasterias polaris, } \\
\text { H. coarctatus, Urticina sp. }\end{array}$ & $\begin{array}{l}\text { Neptunea heros, } \\
\text { Evasterias echinosoma, } \\
\text { C. opilio, } \\
\text { Gorgonocephalus caryi, } \\
\text { Pagurus trigonocheirus }\end{array}$ \\
\hline OD31 & A33 & 9560 & 1312 & 993 & 64 & $\begin{array}{l}\text { Asterias amurensis, } \\
\text { Neocrangon communis/Argis lar, } \\
\text { L. nanimensis, C. opilio, } \\
\text { Styela rustica }\end{array}$ & $\begin{array}{l}\text { A. amurensis, } \\
\text { Styela macrenteron, } \\
\text { L. polaris, Scyphozoa, } \\
\text { L. nanimensis, } \text { N. heros }\end{array}$ \\
\hline OM4-08 & A16 & 70668 & 6272 & 30811 & 208 & $\begin{array}{l}\text { C. opilio, Leptasterias sp., } \\
\text { L. polaris, Ophiura sarsi, } \\
\text { small gastropods }\end{array}$ & $\begin{array}{l}\text { L. polaris, O. sarsi, } \\
\text { C. opilio, Henricia sp., } \\
\text { Pagurus trigonocheirus }\end{array}$ \\
\hline OD22 & A05 & 4077 & 2742 & 1936 & 63 & $\begin{array}{l}\text { C. opilio, Actinostola sp., } \\
\text { L. polaris, Gersemia sp., } \\
\text { undidentified anemone }\end{array}$ & $\begin{array}{l}\text { L. polaris, Stomphia sp., } \\
\text { G. caryi, N. heros, } \\
\text { H. coarctatus }\end{array}$ \\
\hline $\mathrm{R} 17$ & A03 & 17027 & 4393 & 89 & 0 & $\begin{array}{l}\text { Chlamys sp., Stomphia sp., } \\
\text { P. agurus rathbuni, } \\
\text { Neptunea ventricosa, } \\
\text { H. coarctatus }\end{array}$ & $\begin{array}{l}\text { L. polaris, } N \text {. heros, } \\
\text { N. ventricosa, G. caryi, } \\
\text { H. coarctatus }\end{array}$ \\
\hline OD20 & A01 & 5573 & 1688 & 1675 & 72 & $\begin{array}{l}\text { C. opilio, } \mathbf{N} \text {. ventricosa, } \\
\text { P. rathbuni, Actinostola sp., } \\
\text { N. communis/A. lar }\end{array}$ & $\begin{array}{l}\text { G. caryi, L. polaris, N. heros, } \\
N . \text { ventricosa, } \\
\text { P. trigonocheirus }\end{array}$ \\
\hline OM14-08 & A56 & 209080 & 1097 & 111552 & 27 & $\begin{array}{l}\text { C. opilio, O. sarsi, Leptasterias sp., } \\
\text { medium-sized gastropods }\end{array}$ & $\begin{array}{l}\text { L. polaris, G. caryi, C. opilio, } \\
\text { Stomphia coccinea, } \\
\text { Leptasterias sp. }\end{array}$ \\
\hline
\end{tabular}


It is unclear if the body size of Chionoecetes opilio, an important measure of reproductive status and market value in commercial crabs, also changed over the decades in the Chukchi Sea. Studies on size-atmaturity in females demonstrated a smaller size in colder waters at high latitudes and a larger size in warmer temperatures at low latitudes in the Bering and southern Chukchi Seas (Jewett 1981, Orensanz et al. 2007). Our non-quantitative observations and an ongoing study in the northern Bering Sea measured very few large (>35 to $40 \mathrm{~mm}$ carapace width) and mature females occurring north of St. Lawrence Island (Lovvorn 2008), similar to studies in the late 1970s and 1980s in the southeastern and northern Chukchi Sea (Jewett 1981, Frost \& Lowry 1983). The largest male C. opilio caught in the northeastern Chukchi Sea in 1976 was 75 mm (Frost \& Lowry 1983), and only a few males larger than that were caught in the early 1990s (Barber et al. 1997). However, C. opilio as large as 114 $\mathrm{mm}$ were recently caught in the Alaskan Beaufort Sea (H. Chenelot, University of Alaska Fairbanks, pers. obs.), and the species dominated at several stations, though at greater depths than in the Chukchi Sea (Loggerwell \& Rand 2009). Carey (1977), in contrast, did not list $C$. opilio among the dominant species in his Beaufort Sea trawls conducted in the 1970s. To clarify temporal changes and distribution pathways, consistent use of gear types, identification of source areas for snow crab larvae, and environmental limitations for larvae and adults in the Bering, Chukchi and Beaufort Seas are clearly needed.

In terms of temporal changes in community structure, 1 to 3 of the 5 dominant species among the 8 comparable stations pairs were identical between 1976 and the 2000s, which suggests that communities were still similar overall (Table 8). Likewise, 17 yr before Feder et al.'s (2005) sampling, sea stars, brittle stars, decapods and tunicates dominated the southeastern Chukchi Sea epifauna (Sparks \& Pereyra 1966). Some quantitative differences in dominance, however, were obvious. The basket star Gorgonocephalus caryi and the gastropod Neptunea ventricosa (called N. heros in Feder et al. 2005), which were among the 5 dominant species at half of the compared locations in Feder et al. (2005), were absent (G. caryi) or only present at 2 stations ( $N$. ventricosa) among the 5 dominant species in the current study. The snail also had decreased in frequency of occurrence, biomass and abundance in 1998 relative to 1976, while the basket star had not changed frequency of occurrence or biomass, but increased in abundance (Feder et al. 2005). Perhaps the increase in snow crab biomass had sufficiently heightened the competition for food resources, either directly or through indirect food web links, to result in a reduced snail population.

\section{Environmental drivers}

Important factors influencing benthos may differ for biomass and taxonomic composition and will be discussed separately. Benthic biomass, in general, is largely controlled by the supply of food derived from surface waters and reaching the seafloor through pelagic-benthic coupling processes (Graf 1989, Grebmeier \& Barry 1991). Similarly, spatial patterns in macrofaunal biomass in the Chukchi Sea have been linked to variations in pelagic primary production and carbon flux (vertical and advective) to the sea floor under different water masses, namely the ACW and BSAW (Grebmeier et al. 2006a, Lee et al. 2007). In the present study, however, epibenthic abundance and biomass did not differ between these water masses or water mass characteristics. Moreover, epifaunal biomass was not significantly correlated with macrofaunal biomass (Fig. 8), suggesting different processes may be driving observed patterns in these 2 size classes. Interestingly, the 2 indicators of food availability we considered (sediment organic carbon and integrated water column chlorophyll concentration) did not appear to explain differences in epifaunal biomass, whereas food availability variables did correspond well with macrofaunal biomass (Grebmeier et al. 2006a). The lack of correlation to epifaunal biomass might be related to the small number of data points or the use of binned sediment organic carbon data. However, we suspect this difference reflects weaker pelagic-benthic coupling for epibenthic megafauna than for macrofauna (Grebmeier et al. 2006a). Coupling could be weak due to: (1) the higher mobility of many biomass-rich epifaunal organisms obscuring relationships of biomass to food availability at any given location (although sessile epifauna alone were not correlated to these variables either) or (2) the predatory, scavenging, or opportunistic feeding types of many of the biomassdominating species such as Ophiura sarsi (Warner 1982), Chionoecetes opilio (Feder \& Jewett 1981, Squires \& Dawe 2003) and sea stars (Jangoux \& Lawrence 1982), again obscuring pelagic-benthic coupling at any given station. In contrast, several of the biomass-dominating macrofaunal benthic species such as ampeliscid amphipods, macrofaunal clams and various polychaetes (Grebmeier et al. 1989, Feder et al. 2007, Sirenko \& Gagaev 2007) are sessile suspension or filter feeders, directly exploiting fresh organic carbon from the water column (Iken et al. in press).

While none of the environmental variables tested appeared to explain biomass patterns, epibenthic community structure was most strongly related to substrate type and latitude (Table 5). In comparison, sediment 
type along with temperature and salinity structured small demersal fish communities in the Chukchi Sea, while water mass characteristics alone determined the structure and spatial distribution of fish larvae (Norcross et al. in press) and zooplankton communities (Hopcroft et al. in press). Investigations on the East Greenland shelf also suggest coarse substrate is more important for structuring epibenthic communities than the degree of pelagic-benthic coupling or water mass characteristics (Mayer \& Piepenburg 1996). Such differences in community structure between hard or rocky areas and soft muddy areas are not surprising, given the differences in feeding modes suited to these 2 environments. The effects of such differences in the ecology of soft- and hard-substrate environments were obvious in the list of taxa that contributed most to the dissimilarity between the south/southeastern (hard bottom) and other station clusters in the present study. In particular, some sites in the SSE cluster were dominated by Strongylocentrotus pallidus, Hyas coarctatus and various sea stars (Fig. 6, Table 4). Some mobile species such as crabs are thought to succeed in coarse substrate areas because their structural complexity provides shelter for larvae and early juveniles (Tapella et al. 2009). Taxonomic richness was also highest in the south/southeastern station group due to the presence of various sessile taxa that require coarse substrate for attachment (e.g. bryozoans, sponges). Further, coarse substrate occurs in areas with relatively high current velocities, where moving water delivers food resources to these sessile suspensions feeders (Leichter \& Witman 1997).

Patterns in taxonomic composition again contrast with macrofaunal studies on Arctic shelves that indicate greater importance of grain size, sediment $\mathrm{C} / \mathrm{N}$ ratios and porosity (northern Chukchi Sea; Feder et al. 1994b), water depth, temperature and oxygen concentration (Beaufort shelf; Conlan et al. 2008), or surface primary production or surrogates thereof (Laptev Sea; Steffens et al. 2006 and Canada Basin; MacDonald et al. in press). Latitude, which was important in epifaunal community structure in the present study, is likely an indirect indicator of some of these same features (e.g. water masses, primary production), but also suggests the influence of additional factors such as historical zoogeography and seasonal ice cover. Thus, it remains unclear exactly which combination of variables is most important in governing epifaunal community patterns and to what degree.

\section{Conclusions and outlook}

The Chukchi Sea has undergone dramatic changes in the last decades in some environmental character- istics such as ice cover and volume of water transported through the Bering Strait (Stroeve et al. 2005, Woodgate et al. 2006), with anticipated biological consequences. Additional pressures are related to the increased interest in oil exploration in the area. One of the goals of the RUSALCA program and the newly formed Marine Expert Group on the 'Conservation of Arctic Fauna and Flora' (CAFF) under the Arctic Council is to document biological changes and develop monitoring strategies. Our results offer some indications as to which ecosystem components are most in need of monitoring. Several approaches were chosen in other regions, including monitoring of total benthic biomass, e.g. in the St. Lawrence Island polynya in the Bering Sea (Grebmeier et al. 2006b), distribution and/or biomass of either dominant taxa (Zhang \& Tingwei 2003), commercially important taxa, particular 'indicator species' (Carignan \& Villard 2002), or indices of diversity (Noss 1990). For Chukchi Sea epifauna, any measure of benthic biomass, regardless of the target organism(s), can only be a useful climate change indicator if consistent gear is used, ideally including a combination of trawls and photographic methods, which are benign and have high accuracy (Eleftherious \& MacIntyre 2005). Furthermore, our study demonstrates that many dominant epifaunal species are widely distributed across the Chukchi Sea, so that monitoring presence/absence alone will not detect changes; rather, abundance and/or biomass estimates and/or size distributions are needed. Measuring specific aspects of community structure, such as proportions of taxa with different habitat needs (e.g. cold versus warm water) may be most informative. Although recent findings on Chionocetes opilio in the Chukchi Sea suggest that a snow crab fishery is not viable at this point (Feder et al. 2005, Lovvorn 2008, NPFMC 2009, present study), this species should be closely monitored in terms of abundance, biomass, size distribution and reproductive stages, given preliminary evidence of a changing distribution and the potential for future commercial interest. Monitoring changes in the distribution of species that have their northern or southern distribution limits in the Chukchi Sea (e.g. Fig. 7) might also foreshadow ecosystem change. If water temperature is the main factor limiting the distribution of Pacific species to the north, one might expect range expansion in Chukchi Sea species as warming continues (see Sirenko \& Gagaev 2007 for recent possible range extensions in the Chukchi Sea) and a decrease in the abundance of cold-water Arctic species. Species composition and number of taxa along a south-north gradient is informative on the location (and potential change) of the biogeographic boundary of Pacific versus Arctic species. 
Acknowledgements. Vessel operations were supported by the captains and crews of the vessels 'Professor Khromov', 'Oshoru Maru' and 'Oscar Dyson'. We are greatly indebted to chief scientists, for logistical support; to the taxonomic experts, for species identifications; and to many onboard, for assistance with trawl sorting, especially D. Hondolero, P. Drobny, L. Carrothers and J. Richar (all University of Alaska Fairbanks). The present study was funded by the National Oceanographic and Atmospheric Administration through their Cooperative Institute for Alaska Research under Grant No. CIFAR 10-069 and the University of Alaska's Coastal Marine Institute under Grant No. MO7AC12462. B.S. was funded through RFBR Grant No. 04-04-49300, and S.M.H. was supported by an International Polar Year post-doctoral fellowship from University of Alaska Foundation. The RUSALCA Program (2004 cruise), Hokkaido University (OM 2007, 2008) and the BASIS program of the Alaska Fisheries Science Center (OD 2008) contributed the vessel costs for the study collections. We gratefully acknowledge 3 reviewers whose comments improved the article. The present study contributes to the Arctic Ocean Diversity Census of Marine Life project.

\section{LITERATURE CITED}

Alvsvåg J, Agnalt AL, Jørstad KE (2009) Evidence for a permanent establishment of the snow crab (Chionoecetes opilio) in the Barents Sea. Biol Invasions 11:587-595

Ambrose WG Jr, Clough LM, Tilney PR, Beer L (2001) Role of echinoderms in benthic remineralization in the Chukchi Sea. Mar Biol 139:937-949

Anisimova NA (1989) Distributional patterns of echinoderms in the Eurasian sector of the Arctic. In: Herman Y (ed) The Arctic seas. Climatology, oceanography, geology and biology. Van Nostrand Reinhold Company, New York, p 281-301

Aronson RB, Sues HD (1987) The paleo-ecological significance of an anachronistic community. In: Kerfoot WC, Sih A (eds) Predation: direct and indirect impacts on aquatic communities. University Press of New England, Hanover, NH, p 355-366

Barber WE, Smith RL, Vallarino M, Meyer RM (1997) Demersal fish assemblages of the northeastern Chukchi Sea, Alaska. Fish Bull 95:195-209

Billett DSM, Bett BJ, Rice AL, Thurston MH, Galeron J, Sibuet M, Wolff GA (2001) Long-term change in the megabenthos of the Porcupine Abyssal Plain (NE Atlantic). Prog Oceanogr 50:325-348

Bluhm BA, Juterzenka Kv, Piepenburg D (1998) Distribution, standing stock, growth, mortality and production of Strongylocentrotus pallidus (Echinodermata: Echinoidea) in the northern Barents Sea. Polar Biol 20:325-334

Brey T, Rumohr H, Ankar S (1988) Energy content of macrobenthic invertebrates: general conversion factors from weight to energy. J Exp Mar Biol Ecol 117:271-278

Carey AG (1977) Summarization of existing literature and unpublished data on the distribution, abundance, and life histories of benthic organisms (Beaufort Sea). Final Report Contract No 03-5-022-68, Vol I, Outer Continental Shelf Energy Program, NOAA/BLM

Carignan V, Villard MA (2002) Selecting indicator species to monitor ecological integrity, a review. Environ Monit Assess 78:45-61

Clarke KR, Gorley RN (2006) PRIMER v6: user manual/tutorial. PRIMER-E, Plymouth

Clarke KR, Warwick RM (2001) Change in marine communi- ties. An approach to statistical analysis and interpretation, 2nd edn. PRIMER-E, Plymouth

Coachman LK (1987) Advection and mixing on the BeringChukchi Shelves. In: ISHTAR 1986 Progress Report, Vol I, Component A. Advection and mixing of coastal water on high latitude shelves. Institute of Marine Science, University of Alaska, Fairbanks, AK, p 1-42

Coachman LK, Aagaard K, Tripp RB (1975) Bering Strait: the regional physical oceanography. University of Washington Press, Seattle, WA

Conlan K, Aitken A, Hendrycks E, McClelland C, Melling H (2008) Distribution patterns of Canadian Beaufort Shelf macrobenthos. J Mar Syst 74:864-886

Conners ME, Hollowed AB, Brown E (2002) Retrospective analysis of Bering Sea bottom trawl surveys: regime shift and ecosystem reorganization. Proc Oceanogr 55:209-222

Coyle KO, Gillispie JA, Smith RL, Barber WE (1997) Food habits of four demersal Chukchi Sea fishes. Am Fish Soc Symp 19:310-318

Coyle KO, Bluhm BA, Konar B, Blanchard A, Highsmith RC (2007) Amphipod prey of gray whales in the northern Bering Sea: comparison of biomass and distribution between the 1980s and 2002-2003. Deep-Sea Res II 54: 2906-2918

Cross WE, Thomson DH (1987) Effects of experimental releases of oil and dispersed oil on Arctic nearshore macrobenthos. I. Infauna. Arctic 40(Supp 1):184-200

Dahm C (1996) Ecology and population dynamics of Antarctic ophiuroids (Echinodermata). Rep Polar Res 194:1-289 (in German)

Deubel H (2000) Structures and nutrition requirements of macrozoobenthic communities in the area of the Lomonossov Ridge in the Arctic Ocean. Rep Polar Res 370: 1-147 (in Germany)

Deubel H, Engel M, Fetzer I, Gagaev S and others (2003) The southern Kara Sea ecosystem: phytoplankton, zooplankton and benthos communities influenced by river run-off. In: Stein R, Fahl K, Fuetterer DK, Galimov EM, Stepanets OV (eds) Siberian river run-off in the Kara Sea. Elsevier, Amsterdam, p 237-275

> Dunton KH, Goodall JL, Schonberg SV, Grebmeier JM, Maidment DR (2005) Multi-decadal synthesis of benthic-pelagic coupling in the western Arctic: role of cross-shelf advective processes. Deep-Sea Res II 52:3462-3477

Eleftherious A, MacIntyre AC (2005) Methods for study of marine benthos. Blackwell Publishers, Oxford

ESRI (Environmental Systems Research Institute) (2005) ArcGIS Desktop v.9.1; available at: www.esri.com/software/ arcgis/

Fair FL, Nelson A (1999) Southeast Chukchi Sea and Kotzebue Sound trawl survey, 1998. Regional Information Report No 3A99-34, Alaska Department of Fish \& Game Regional Information Report. Anchorage, AK, p 1-106

Feder HM, Jewett SC (1978) Survey of the epifaunal invertebrates of Norton Sound, southeastern Chukchi Sea, and Kotzebue Sound. IMS Report R78-1, Institute of Marine Science, University of Alaska Fairbanks, Fairbanks, AK, p 1-124

Feder HM, Jewett SC (1980) A survey of the epifaunal invertebrates of the southeastern Bering Sea with notes on the feeding biology of selected species. IMS Report R78-5, Institute of Marine Science, University of Alaska Fairbanks, Fairbanks, AK, p 1-105

Feder HM, Jewett SC (1981) Feeding interactions in the eastern Bering Sea with emphasis on the benthos. In: Hood DW, Calder JA (eds) The eastern Bering Sea shelf: oceanography and resources, Vol II. NOAA, University 
Press, Seattle, WA, p 1229-1261

Feder HM, Foster NR, Jewett SC, Weingartner TJ, Baxter R (1994a) Mollusks in the northeastern Chukchi Sea. Arctic 47:145-163

Feder HM, Naidu AS, Jewett SC, Hameedi JM, Johnson WR, Whitledge TE (1994b) The northeastern Chukchi Sea: benthos-environmental interactions. Mar Ecol Prog Ser 111:171-190

Feder HM, Jewett SC, Blanchard A (2005) Southeastern Chukchi Sea (Alaska) epibenthos. Polar Biol 28:402-421

Feder HM, Jewett SC, Blanchard AL (2007) Southeastern Chukchi Sea (Alaska) macrobenthos. Polar Biol 30: 261-275

Federal Register (2007) Outer Continental Shelf (OCS), Alaska OCS Region, Beaufort Sea and Chukchi Sea, proposed oil and gas lease sales for years 2007 to 2012. Federal Register Vol 2, no. 179 (microfiche)

Foyle TP, O'Dor RK, Elner RW (1989) Energetically defining the thermal limits of the snow crab. J Exp Biol 145: 371-393

Frederich M, Sartoris FJ, Arntz WE, Pörtner HO (2000) Haemolymph $\mathrm{Mg}^{2+}$ regulation in decapod crustaceans: physiological correlates and ecological consequences in polar areas. J Exp Biol 203:1383-1393

Frost KJ, Lowry LF (1983) Demersal fishes and invertebrates trawled in the northeastern Chuckhi and western Beaufort Seas, 1976-77. NOAA Tech Rep 764:1-22

Fujita T, Ohta S (1989) Spatial structure within a dense bed of the brittle star Ophiura sarsi (Ophiuroidea: Echinodermata) in the bathyal zone off Otsuchi, northeastern Japan. J Oceanogr Soc Jpn 45:289-300

$>$ Graf G (1989) Benthic-pelagic coupling in a deep-sea benthic community. Nature 341:437-439

Grebmeier JM (1993) Studies of pelagic-benthic coupling extended onto the Soviet continental shelf in the northern Bering and Chukchi Seas. Cont Shelf Res 13:653-668

> Grebmeier JM, Barry JP (1991) The influence of oceanographic processes on pelagic-benthic coupling in polar regions: a benthic perspective. J Mar Syst 2:495-518

Grebmeier JM, McRoy CP, Feder HM (1988) Pelagic-benthic coupling on the shelf of the northern Bering and Chukchi Seas. I. Food supply source and benthic biomass. Mar Ecol Prog Ser 48:57-67

Grebmeier JM, Feder HM, McRoy CP (1989) Pelagic-benthic coupling on the shelf of the northern Bering and Chukchi seas. 2. Benthic community structure. Mar Ecol Prog Ser 51:253-268

Grebmeier JM, Cooper LW, Feder HM, Sirenko BI (2006a) Ecosystem dynamics of the Pacific-influenced northern Bering and Chukchi Seas in the Amerasian Arctic. Prog Oceanogr 71:331-361

Grebmeier JM, Overland JE, Moore SE, Farley EV and others (2006b) A major ecosystem shift in the northern Bering Sea. Science 311:1461-1463

> Gunderson DR, Ellis IE (1986) Development of a plumb staff beam trawl for sampling demersal fauna. Fish Res 4:35-41

Hamazaki T, Fair L, Watson L, Brennan E (2005) Analyses of Bering Sea bottom-trawl surveys in Norton Sound: absence of regime shift effect on epifauna and demersal fish. ICES J Mar Sci 62:1597-1602

Highsmith RC, Coyle KO (1990) High productivity of northern Bering Sea benthic amphipods. Nature 344:862-864

Highsmith RC, Coyle KO (1992) Productivity of arctic amphipods relative to gray whale energy requirements. Mar Ecol Prog Ser 83:141-150

> Hopcroft RR, Kosobokova KN, Pinchuk AI (in press) Zooplankton community patterns in the Chukchi Sea during summer 2004. Deep-Sea Res II doi:10.1016/j.dsr2.2009.08. 003

Iken K, Brey T, Wand U, Voigt J, Junghans P (2001) Food web structure of the benthic community at the Porcupine Abyssal Plain (NE Atlantic): a stable isotope analysis. Prog Oceanogr 50:383-405

Iken K, Bluhm BA, Dunton K (in press) Benthic food-web structure serves under differing water mass properties in the southern Chukchi Sea. Deep-Sea Res II doi: 10.1016/ j.dsr2.2009.08.007

Jangoux M, Lawrence JM (1982) Echinoderm nutrition. AA Balkema, Rotterdam

Jewett SC (1981) Variations in some reproductive aspects of female snow crabs Chionoecetes opilio. J Shellfish Res 1:95-99

Jewett SC, Feder HM (1981) Epifaunal invertebrates of the continental shelf of the eastern Bering and Chukchi Seas. In: Hood DW, Calder JA (eds) The eastern Bering Sea shelf: oceanography and resources, Vol II. University of Washington Press, Seattle, WA, p 1131-1153

Kuzmin SA, Akhtarin SM, Menis DT (1998) The first finding of snow crab Chionoecetes opilio (Decapoda, Majidae) in the Barents Sea. Zool Zh 77:489-491

Lee SH, Whitledge TE, Kan SH (2007) Recent carbon and nitrogen update rates of phytoplankton in Bering Strait and the Chukchi Sea. Cont Shelf Res 27:2231-2249

> Leichter JJ, Witman JD (1997) Water flow over subtidal rock walls: relation to distributions and growth rates of sessile suspension feeders in the Gulf of Maine water flow. J Exp Mar Biol Ecol 209:293-307

Loggerwell L, Rand K (2009) Beaufort Sea marine fish monitoring 2008: pilot survey and test of hypotheses. Final report OCS Study MMS 2008-062, Minerals Management Service, Anchorage, AK

Lovvorn JR (2008) Predicting snow crab growth and size with climate warming in the northern Bering Sea. North Pacific Research Board Project 713, Semiannual reports January and July 2008; available at: http://project.nprb.org/view. jsp?id=20b711d5-b140-4a48-af2a-566943b7339e

Lovvorn JR, Richman SE, Grebmeier JM, Cooper LW (2003) Diet and body condition of spectacled eiders wintering in pack ice of the Bering Sea. Polar Biol 26:259-267

> Ma Y, Zhang MY, Cui T (2003) Preliminary research on dominant species identification of red tide organisms by airborne hyperspectral technique. Proc SPIE 4892:278-286

- MacDonald IR, Bluhm B, Iken K, Gagaev S, Strong S (in press) Benthic macrofauna and megafauna assemblages in the Arctic deep-sea Canada Basin. Deep-Sea Res II doi: 10.1016/j.dsr2.2009.08.012

MacGinitie GE (1955) Distribution and ecology of the invertebrates of Point Barrow, Alaska. Smithson Misc Collect 128:1-201

MacGinitie N (1959) Marine Mollusca of Point Barrow, Alaska. Proc US Natl Mus 109:59-208

Mayer M, Piepenburg D (1996) Epibenthic community patterns on the continental slope off East Greenland at $75^{\circ} \mathrm{N}$. Mar Ecol Prog Ser 143:151-164

Mecklenburg CW, Stein DL, Sheiko B, Chernova NV, Mecklenburg TA, Holladay BA (2007) Russian-American longterm census of the Arctic: benthic fishes trawled in the Chukchi Sea and Bering Strait, August 2004. Northwest Nat 88:168-187

Metaxas A, Griffin B (2004) Dense beds of the ophiuroid Ophiacantha abyssicola on the continental slope off Nova Scotia, Canada. Deep-Sea Res I 51:1307-1317

> Moore SE, Grebmeier JM, Davies JR (2003) Gray whale distribution relative to forage habitat in the northern Bering 
Sea: current conditions and retrospective summary. Can J Zool 81:734-742

Morton B, Jones DS (2003) The dietary preferences of a suite of carrion-scavenging gastropods (Nassariidae, Buccinidae) in Princess Royal Harbour, Albany, Western Australia. J Mollusc Stud 69:151-156

Naidu AS (1988) Marine surficial sediments, Section 1.4. In: Ehler CN, Basta EJ, LaPointe CF, Ray GC (eds) Bering, Chukchi and Beaufort Seas: coastal and ocean zones strategic assessment data atlas. NOAA Strategic Assessment Branch, Ocean Assessment Division, Washington, DC

Norcross BL, Holladay BA, Busby MS, Mier KL (in press) Demersal and larval fish assemblages in the Chukchi Sea. Deep-Sea Res II doi: 10.1016/j.dsr2.2009.08.006

Noss RF (1990) Indicators for monitoring biodiversity: a hierarchical approach. Conserv Biol 4:355-364

NPFMC (North Pacific Fisheries Management Council) (2009) Public review draft-Fishery management plan for rish resources of the Arctic management area. Available at: www.fakr.noaa.gov/npfmc/current_issues/Arctic/Arctic EA109.pdf (accessed 15 May 2009)

Oliver JS, Slattery PN, O'Connor EF, Lowry LF (1983) Walrus, Odobenus rosmarus, feeding in the Bering Sea: a benthic perspective. Fish Bull 81:501-512

Orensanz J, Ernst B, Armstrong DA, Stabeno P, Livingston P (2004) Contraction of the geographic range of distribution of snow crab (Chionoecetes opilio) in the eastern Bering Sea: An environmental ratchet? CalCOFI Rep 44:65-79

> Orensanz JM, Ernst B, Armstrong DA (2007) Variation of female size and stage at maturity in snow crab (Chionoecetes opilio) (Brachyura: Majidae) from the eastern Bering Sea. J Crustac Biol 27:576-591

Paul JM, Paul AJ, Barber WE (1997) Reproductive biology and distribution of the snow crab from the northeastern Chukchi Sea. Am Fish Soc Symp 19:287-294

Pickart RS (2006) RUSALCA Joint Bering/Chukchi Sea Expedition 9-24 August 2004: CTD data processing report. Available at: www.whoi.edu/science/PO/people/pickart/reports/ Khromov_Report_Mod.pdf, www.whoi.edu/science/PO/ pickart/plots/khromov_plots.htm (accessed 18 April 2009)

Pickart RS, Weingartner TJ, Pratt LJ, Zimmermann S, Torres DJ (2005) Flow of winter-transformed Pacific water into the western Arctic. Deep-Sea Res II 52:3175-3198

Pickart RS, Pratt LJ, Torres DJ, Whitledge TE and others (in press) Evolution and dynamics of the flow through Herald Canyon in the western Chukchi Sea. Deep-Sea Res II doi: 10.1016/j.dsr2.2009.08.002

Piepenburg D (2000) Arctic brittle stars (Echinodermata: Ophiuroidea). Oceanogr Mar Biol Annu Rev 38:189-256

Piepenburg D, Brandt A, Juterzenka Kv, Mayer M, Schnack K, Seiler D, Witte U, Spindler M (2001) Patterns and determinants of the distribution and structure of benthic faunal assemblages in the northern North Atlantic. In: Schäfer P, Ritzrau W, Schlüter M, Thiede T (eds) The northern North Atlantic: a changing environment. Springer, Berlin, p 179-198

> Piepenburg D, Schmid MK (1996a) Distribution, abundance, biomass, and mineralization potential of the epibenthic megafauna of the northeast Greenland shelf. Mar Biol 125:321-332

Piepenburg D, Schmid MK (1996b) Brittle star fauna (Echinodermata: Ophiuroidea) of the Arctic northwestern Barents Sea: composition, abundance, biomass and spatial distribution. Polar Biol 16:383-392

Piepenburg D, Schmid MK (1997) A photographic survey of the epibenthic megafauna of the Arctic Laptev Sea shelf: distribution, abundance, and estimates of biomass and organic carbon. Mar Ecol Prog Ser 147:63-75

> Piepenburg D, Blackburn TH, von Dorrien CF, Gutt J and others (1995) Partitioning of benthic community respiration in the Arctic (northwestern Barents Sea). Mar Ecol Prog Ser 118:199-213

Piepenburg D, Chernova NV, von Dorrien CF, Gutt J and others (1996) Megabenthic communities in the waters around Svalbard. Polar Biol 16:431-446

$>$ Piepenburg D, Ambrose WG, Brandt A, Renaud PE, Ahrens MJ, Jensen P (1997) Benthic community patterns reflect water column processes in the Northeast Water polynya (Greenland). J Mar Syst 10:467-482

Rodionov S, Overland JE (2005) Application of a sequential regime shift detection method to the Bering Sea ecosystem. ICES J Mar Sci 62:328-332

Rowe GT (1983) Biomass and production of the deep-sea macrobenthos. In: Rowe GT (ed) The sea, Vol 8. Deep-sea biology. John Wiley and Sons, New York, p 97-121

- Sejr MK, Jensen KT, Rysgaard S (2000) Macrozoobenthic community structure in a high-arctic East Greenland fjord. Polar Biol 23:792-801

Sirenko BI, Gagaev SY (2007) Unusual abundance of macrobenthos and biological invasions in the Chukchi Sea. Russ J Mar Biol 33:355-364

Sparks AK, Pereyra WT (1966) Benthic Invertebrates of the Southeastern Chukchi Sea. In: Wilimovsky NJ, Wolfe JN (eds) Environment of the Cape Thompson region, Alaska. pp. 817-838. US Atomic Energy Commission Division of Technical Information Extension, Oak Ridge, TN

- Springer AM, McRoy CP (1993) The paradox of pelagic food webs in the northern Bering Sea. III. Patterns of primary production. Cont Shelf Res 13:575-599

Springer A, McRoy CP, Flint M (1996) The Bering Sea Green Belt: shelf-edge processes and ecosystem production. Fish Oceanogr 5:205-223

> Squires HJ, Dawe EG (2003) Stomach contents of snow crab (Chionoecetes opilio, Decapoda, Brachyura) from the northeast Newfoundland shelf. J Northwest Atl Fish Sci $32: 27-38$

Starmans A, Gutt J, Arntz WE (1999) Mega-epibenthic communities in Arctic and Antarctic shelf areas. Mar Biol 135: 269-280

> Steffens M, Piepenburg D, Schmid MK (2006) Distribution and structure of macrobenthic fauna in the eastern Laptev Sea in relation to environmental factors. Polar Biol 29: 837-848

> Stroeve JC, Serreze MC, Fetterer F, Arbetter T, Meier W, Maslanik J, Knowles K (2005) Tracking the Arctic's shrinking ice cover: another extreme September minimum in 2004. Geophys Res Lett 32:L04501 doi:10.1029/ 2004GL021810

Tapella F, Romera MC, Stevens BG, Buck CL (2009) Substrate preferences and redistribution of blue kind crab Paralithodes platypus glaucothoe and first crab on natural substrates in the laboratory. J Exp Mar Biol Ecol 372:31-35

Taylor DM, O'Keefe PG (1986) Analysis of the snow crab, Chionoecetes opilio, fishery in Newfoundland in 1985. Can Atlantic Fish Sci Advisory Committee Res Document 86/57, Canadian Science Advisory Secretariat, Ottawa

Thatje S, Arntz WE (2004) Antarctic reptant decapods: More than a myth? Polar Biol 27:195-201

Ushakov PV (1952) Chukchi Sea and its bottom fauna. Krainii severo-vostok Soyuza SSR. Fauna I flora Chukotskogo moray (Extreme southeast of the USSR, fauna and flora of the Chukchi Sea), vol 2, p 5-83 (in Russian)

- Walsh JJ, McRoy CP, Coachman LK, Goering JJ and others (1989) Carbon and nitrogen cycling within the Bering/ 
Chukchi Seas: source regions for organic matter affecting AOU demands of the Arctic Ocean. Prog Oceanogr 22: 277-359

Walsh JJ, Dieterls DA, Maslowski W, Grebmeier JM and others (2005) A numerical model of neglected spring primary production within the Chukchi/Beaufort Seas. Deep-Sea Res II 52:3541-3576

Warner GF (1982) Food and feeding mechanisms: Ophiuroidea. In: Jangoux M, Lawrence JM (eds) Echinoderm nutrition. Balkema, Rotterdam, p 161-181

Weingartner TJ, Danielson S, Sasaki Y, Pavlov V, Kulakov M (1999) The Siberian Coastal Current: a wind- and buoyancy-forced Arctic coast current. J Geophys Res 104: 26697-26713
Weingartner T, Aagaard K, Woodgate R, Danielson S, Sasaki Y, Cavalieri D (2005) Circulation on the north central Chukchi Sea shelf. Deep-Sea Res II 52:3150-3174

Wildish DJ, Akagi HM, McKeown DL, Pohle GW (2008) Pockmarks influence benthic communities in Passamaquoddy Bay, Bay of Fundy, Canada. Mar Ecol Prog Ser 357:51-66

Winsor P, Chapman DC (2004) Pathways of Pacific water across the Chukchi Sea: a numerical model study. J Geophys Res 109(C03002) doi: 1029/2003JC001962

Woodgate RA, Aagaard K, Weingartner TJ (2006) Interannual changes in the Bering Strait fluxes of volume, heat and freshwater between 1991 and 2004. Geophys Res Lett 33 doi:10.1029/2006GL026931

Appendix 1. List of epifaunal taxa found during 3 cruises to the Chukchi Sea (2004 to 2007). Taxon identifications were by the authors and by the following taxonomists: Ole Tendal (Zoological Museum Copenhagen, Denmark; Porifera), Daphne Fautin (University of Kansas, Lawrence, USA; Actinaria), Estefania Rodriguez (Ohio State University, Columbus, USA; Actinaria), Ken Coyle (University of Alaska Fairbanks, USA; Amphipoda), Igor Smirnov (Zoological Institute, St. Petersburg, Russia; Ophiuroidea), Christopher Mah (Smithsonian Institution, Washington, DC, USA; Asteroidea), Andrey Gebruk (PP Shirshov, Moscow, Russia; Holothuroidea), Piotr Kuklinski (Institute of Oceanology, Sopot, Poland; Bryozoa) and Linda Cole (Smithsonian Institution, Washington, DC, USA; Ascidiacea)

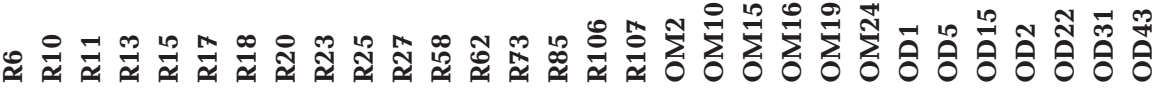

\section{Ascidiacea}

Ascidian, solitary sp. 1

Boltenia echinata

Boltenia ovifera

Chelyosoma macleayanum

Corella cf. inflate

Cystodytes sp.

Dendrodoa aggregate (?)

Didemnum albidum

Diplosoma cf. listerianum

Halocynthia aurantium

Molgula retortiformis

Styela rustica

Synascidian, brown

Synoicum sp.

\section{Cnidaria}

Actiniidae sp. 2

Actinostola sp.

Actinostolidae

Anemone sp. 1

Anemone sp. 2

Anemone sp. 3

Cribrinopsis/Epiactis sp.

Gersemia sp. (cf. rubiformis)

Haliatiidae

Metridium facimen

Stomphia sp.

Urticina sp. 1

Urticina sp.?

Hydrozoa

Crustacea

Acanthostepheria behringiensis

Anonyx sp.

Eusirus cuspidatus

Hippomedon sp.

Lembos arcticus

Melita dentata

Monoculodes sp.

Other amphipods

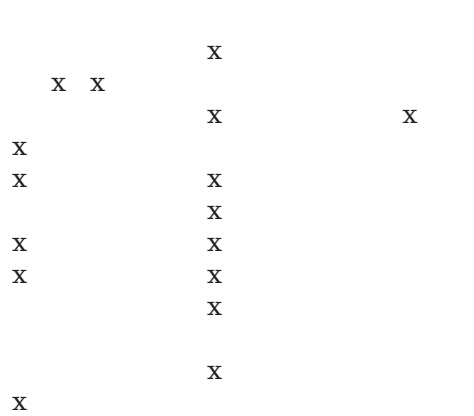

$\mathrm{X}$
$\mathrm{X}$

$\mathrm{x} \quad \mathrm{X}$
$\mathrm{x}$

$\mathrm{x} \quad \mathrm{x}$

x

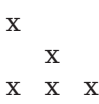

$\mathrm{x}$

$\mathrm{x}$

$\mathrm{x}$

$\mathrm{x}$

$\mathrm{x}$

$\mathrm{x} \quad \mathrm{x}$

$\mathrm{x} \quad \mathrm{X}$

$\mathrm{x} \quad \mathrm{X}$

$\begin{array}{llllllllllll} & x & & x & x\end{array}$

$\mathrm{x}$

$\mathrm{x}$

$\begin{array}{ll}x & x\end{array}$

$\begin{array}{lllll}x & x & x & x & x\end{array}$

$\mathrm{x} \quad \mathrm{x} \quad \mathrm{x}$

$\begin{array}{lll} & \mathrm{X} \\ \mathrm{x} & \mathrm{X}\end{array}$

$x$

$\begin{array}{llll}\mathrm{x} & \mathrm{x} & \mathrm{x} & \mathrm{x}\end{array}$

$\begin{array}{ll}\mathrm{x} & \mathrm{X} \\ \mathrm{x}\end{array}$

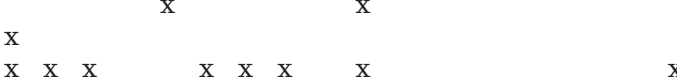

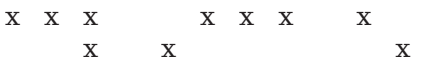

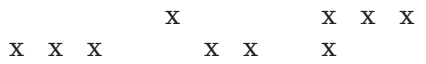

$\mathrm{x} \quad \mathrm{x}$

$\mathrm{x}$$$
\mathrm{x}
$$$$
\mathrm{x} \quad \mathrm{x}
$$

$\mathrm{x} \quad \mathrm{x}$

$\begin{array}{lll}x & x & x\end{array}$

$\mathrm{x}$

$\mathrm{x}$

$\mathrm{x}$ 
Appendix 1 (continued)

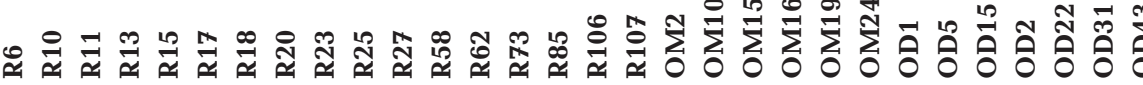

Paramphithoe polyacantha

Paroediceros spp.

Rhachotropis aculeata

Stegocephalidae (incl. S. inflatus)

Stenothoidae

Cirripedia

Chionoecetes opilio

Hyas coarctatus

Labidochirus splendescens

Paguridae sp. 1

Pagurus capillatus (?)

Pagurus rathbuni

Paralithodes platypus

Telmessus cheiragonus

Isopoda

Argis lar \& Neocrangon communis

Other shrimps excl. Pandalidae

Sabinea septemcarinata

Saduria sp.

Sclerocrangon sp.

Spirontocaris sp. (incl S. arcuata)

Pycnogonida

Echinodermata

Asterias amurensis/rathbuni

Crossaster papposum

Ctenodiscus crispatus

Henricia tumida (?)

Leptasterias groenlandica

Leptasterias polaris

Lethasterias nanimensis

Pteraster cf. tesselatus

Pteraster obscurus

Solaster cf. dawsoni

Urasterias linki

Myriotrochus rinkii

Ocnus sp.

Psolus fabricii

Psolus phantapus

Gorgonocephalus caryi

Amphiodia craterodmeta

Ophiacantha sp.

Ophiura sarsi

Stegophiura nodosa

Strongylocentrotus pallidus

Strongylocentrotus sp.

Echinarachnius parma

Mollusca

Amicula vestita

Leptochiton aff. assimilis \&

Stenosemus albus

Admete viridula

Anomolosipho conulus

Boreoscala groenlandica

Boreotrophon clathratus

Buccinum angulosum

Buccinum elatior

Buccinum spp. (incl. B. ciliatum,

B. plectrum, B. polaris,

B. polium obsoletum)

Colus spp.

Cryptonatica affinis

Gastropod sp. 1

Gastropod sp. 3

Gastropod sp. 2

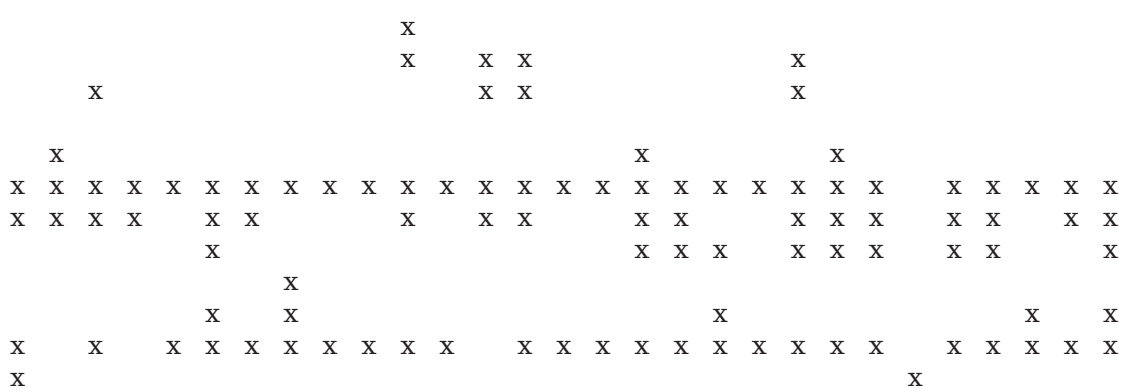

$\mathrm{x}$

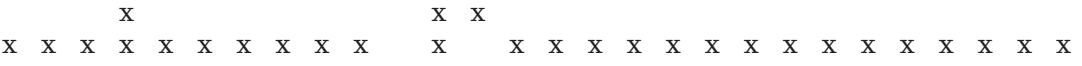
$\begin{array}{lllllllllllllll} & \mathrm{X} & \mathrm{X} & \mathrm{X} & \mathrm{X}\end{array}$

$\mathrm{X}$

$\mathrm{x} \quad \mathrm{x} \quad \mathrm{x}$

$\begin{array}{lllllllllll} & \mathrm{X} & \mathrm{x} & \mathrm{X}\end{array}$

$\mathrm{X} \quad \mathrm{X} \quad \mathrm{x}$

$\mathrm{x} \quad \mathrm{X}$

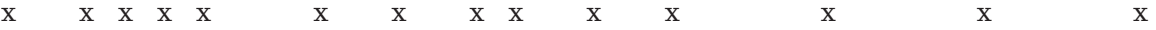

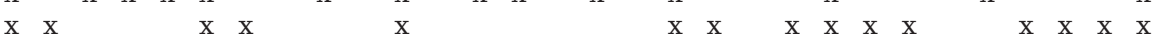

$\begin{array}{llllllllllllllllllll}\mathrm{x} & \mathrm{x} & \mathrm{x} & \mathrm{x} & \mathrm{x} & \mathrm{x} & \mathrm{x} & \mathrm{x} & \mathrm{x} & \mathrm{x} & \mathrm{x} & \mathrm{x} & \mathrm{x} & \mathrm{x} & \mathrm{x} & \mathrm{x} & \mathrm{x} & & \mathrm{x} \\ \mathrm{x} & & & & & & & & & & & & \mathrm{x} & & & & & \mathrm{x} & \mathrm{x}\end{array}$
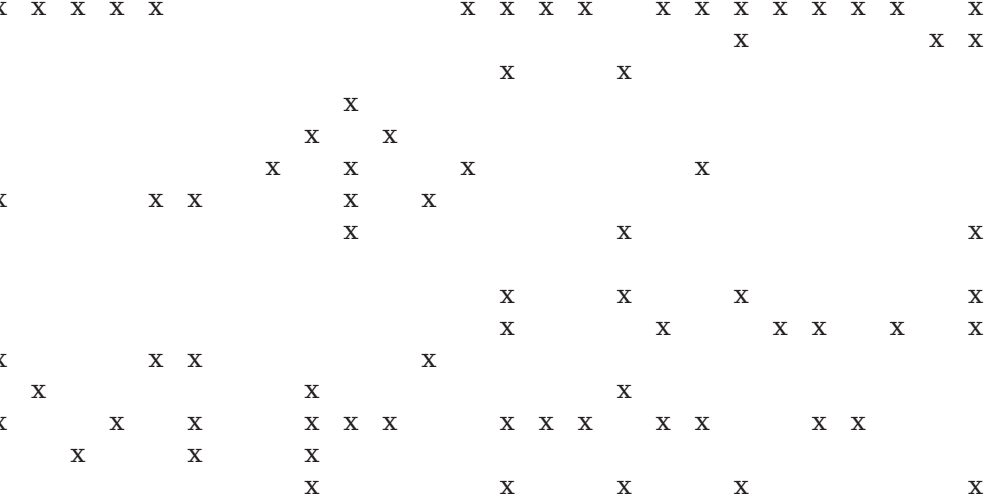

$\mathrm{x} \quad \mathrm{X}$

$\mathrm{X} \times \mathrm{X}$

X X

$\mathrm{X}$

$\mathrm{X} \quad \mathrm{X}$

$\mathrm{x} \quad \mathrm{x}$

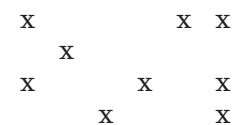

$\mathrm{X} \quad \mathrm{X}$

X

$\mathrm{X}$

$\mathrm{x}$

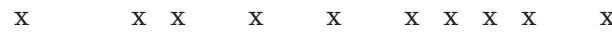

$$
\begin{aligned}
& \mathrm{X}
\end{aligned}
$$

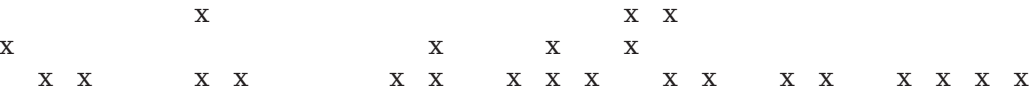

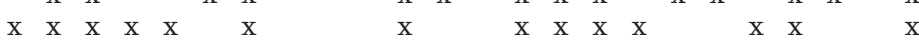

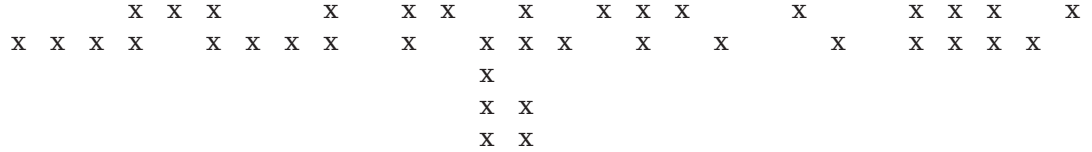


Appendix 1 (continued)

\begin{tabular}{|c|c|c|c|c|c|c|c|c|c|c|c|c|c|c|c|c|c|c|c|c|c|c|c|c|c|c|c|c|}
\hline & $\mathscr{\mathscr { q }}$ & $\stackrel{\circ}{\vec{\sim}}$ & $\overline{\vec{\approx}}$ & $\stackrel{m}{\pi}$ & $\frac{n}{\pi}$ & $\frac{\pi}{a}$ & $\stackrel{\infty}{\pi}$ & $\underset{\widetilde{\pi}}{\approx}$ & $\stackrel{\dddot{\pi}}{\approx}$ & $\stackrel{\mathscr{2}}{\approx}$ & & $\begin{array}{l}\infty \\
\infty \\
\sim\end{array}$ & ֶै & & $\stackrel{\mathscr{2}}{\mathscr{\infty}}$ & & $\frac{\pi}{a}$ & $\sum_{0}^{\mathfrak{y}}$ & & $\sum_{0}^{n}$ & $\sum_{0}^{0}$ & & $\sum_{0}^{J}$ & วิ & $\frac{10}{0}$ & & & $\begin{array}{l}\stackrel{9}{\sharp} \\
\stackrel{7}{0}\end{array}$ \\
\hline Latisipho hypolispus & & & & & & & & $\mathrm{x}$ & & & & & & & & & $\mathrm{x}$ & & & & & & $\mathrm{x}$ & & & $\mathrm{x}$ & & \\
\hline Limpet sp. 1 & & $\mathrm{x}$ & & & & & & & & & & & $\mathrm{x}$ & & & & & & & & & & & & & & & \\
\hline Limpet sp. 2 & & & & & & & & & & & & & $\mathrm{x}$ & & & & & & & & & & & & & & & \\
\hline Lunatia pallida & & & & & $\mathrm{x}$ & & & $\mathrm{x}$ & $\mathrm{x}$ & $\mathrm{x}$ & & $\mathrm{x}$ & & & $\mathrm{x}$ & $\mathrm{x}$ & $\mathrm{x}$ & $\mathrm{x}$ & $\mathrm{x}$ & & & & & & & & $\mathrm{x}$ & \\
\hline Neptunea beringia & & & & & & $\mathrm{x}$ & & & & & & & & & & & & $\mathrm{x}$ & & & & & & & & & & \\
\hline Neptunea communis & & $\mathrm{x}$ & $\mathrm{x}$ & $\mathrm{x}$ & $\mathrm{x}$ & & $\mathrm{x}$ & $\mathrm{x}$ & & & & $\mathrm{x}$ & & & $\mathrm{x}$ & $\mathrm{x}$ & $\mathrm{x}$ & $\mathrm{x}$ & & $\mathrm{x}$ & $\mathrm{x}$ & $\mathrm{x}$ & $\mathrm{x}$ & & & $\mathrm{x}$ & & $\mathrm{x}$ \\
\hline Neptunea lirata & & & & & & $\mathrm{x}$ & & & & & & & & & & & & $\mathrm{x}$ & $\mathrm{x}$ & & $\mathrm{x}$ & & & & $\mathrm{x}$ & $\mathrm{x}$ & & \\
\hline Neptunea ventricosa & & & $\mathrm{x}$ & & $\mathrm{x}$ & $\mathrm{x}$ & $\mathrm{x}$ & & & & & & $\mathrm{x}$ & & $\mathrm{x}$ & & $\mathrm{x}$ & & & $\mathrm{x}$ & & $\mathrm{x}$ & $\mathrm{x}$ & $\mathrm{x}$ & $\mathrm{x}$ & $\mathrm{x}$ & & \\
\hline Nodulotrophon coronatus & & & & & & & & & & & & & & & & & & & & & & & $\mathrm{x}$ & & & & & \\
\hline Nudibranchia & & & $\mathrm{x}$ & $\mathrm{x}$ & & & & & & & & & $\mathrm{x}$ & $\mathrm{x}$ & & & & & & & $\mathrm{x}$ & $\mathrm{x}$ & & & & & & $\mathrm{x}$ \\
\hline Oenopatina sp. & & & & & & & $\mathrm{x}$ & $\mathrm{x}$ & $\mathrm{x}$ & & & $\mathrm{x}$ & & $\mathrm{x}$ & $\mathrm{x}$ & & & $\mathrm{x}$ & & & & & & & & & & \\
\hline Plicifusus kroyeri & & & & & & & & & & & & & & & & & & & & & & & $\mathrm{x}$ & & & & & \\
\hline Plicifusus sp. & & & & & & & & & & & & & & & & & & & & & & & & & $\mathrm{x}$ & & & \\
\hline Propebela nobilis & & & & & & & & & & & & & & & & & & & & $\mathrm{x}$ & & & $\mathrm{x}$ & & & & & \\
\hline Pyrulofusus deformis & & & & & & & & & & & & & & & & & & $\mathrm{x}$ & & & & & & & & & & \\
\hline Silichna alba & & & & & & & & & $\mathrm{x}$ & $\mathrm{x}$ & & & & & & & & & & & & & & & & & & \\
\hline Solariella costalis \& $S$. varicosa & & $\mathrm{x}$ & & $\mathrm{x}$ & $\mathrm{x}$ & $\mathrm{x}$ & $\mathrm{x}$ & $\mathrm{x}$ & $\mathrm{x}$ & $\mathrm{x}$ & $\mathrm{x}$ & $\mathrm{x}$ & $\mathrm{x}$ & $\mathrm{x}$ & $\mathrm{x}$ & & $\mathrm{x}$ & $\mathrm{x}$ & $\mathrm{x}$ & $\mathrm{x}$ & $\mathrm{x}$ & & $\mathrm{x}$ & & & $\mathrm{x}$ & & \\
\hline Tachyrhynchus erosus & & & & $\mathrm{x}$ & & & $\mathrm{x}$ & $\mathrm{x}$ & & & & $\mathrm{x}$ & & $\mathrm{x}$ & $\mathrm{x}$ & $\mathrm{x}$ & $\mathrm{x}$ & $\mathrm{x}$ & $\mathrm{x}$ & $\mathrm{x}$ & & & $\mathrm{x}$ & $\mathrm{x}$ & $\mathrm{x}$ & $\mathrm{x}$ & & \\
\hline Trichotropis bicarinata & & & & $\mathrm{x}$ & & $\mathrm{x}$ & & & & & & & & $\mathrm{x}$ & $\mathrm{x}$ & & & & & $\mathrm{x}$ & & & & & & & & \\
\hline Trichotropis carinata & & & $\mathrm{x}$ & $\mathrm{x}$ & $\mathrm{x}$ & & & $\mathrm{x}$ & & & & & & $\mathrm{x}$ & $\mathrm{x}$ & & & & & & & & & & & & & \\
\hline Velutina sp. & & & & & & & & & & & & & $\mathrm{x}$ & & & & & & & & & & & & & & & \\
\hline Volutopsis sp. & & & & & & & & $\mathrm{x}$ & & & & & & & & & & & & & & & & & & & & \\
\hline Chlamys sp. & & & & & & $\mathrm{x}$ & & & & & & & & & & & & & & & $\mathrm{x}$ & & & & & & & \\
\hline Pododesmus macrochisma & & & & & & & & & & & & & & & & & & & & & & & & & & & & \\
\hline Serripes groenlandicus & & & $\mathrm{x}$ & & $\mathrm{x}$ & & & $\mathrm{x}$ & $\mathrm{x}$ & $\mathrm{x}$ & & & & & & $\mathrm{x}$ & & $\mathrm{x}$ & $\mathrm{x}$ & $\mathrm{x}$ & $\mathrm{x}$ & & $\mathrm{x}$ & & & $\mathrm{x}$ & & \\
\hline Octopus & & & & & & & & & & & & & & & $\mathrm{x}$ & & & & & & & & & & & & & \\
\hline Echiura & & & & $\mathrm{x}$ & $\mathrm{x}$ & & & & & & & & & & & & & & & & & & & & & & & \\
\hline Nemertini & & & $\mathrm{x}$ & $\mathrm{x}$ & $\mathrm{x}$ & & & & $\mathrm{x}$ & $\mathrm{x}$ & & & & & & & & & & & $\mathrm{x}$ & & & & & & & \\
\hline Priapulida & & & & $\mathrm{x}$ & & & & & & & & & & & & & & & & & & & & & & & & \\
\hline Sipunculida & & $\mathrm{x}$ & & & & & & & & & & & & & $\mathrm{x}$ & & & & & & $\mathrm{x}$ & & 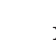 & $\mathrm{x}$ & & & & \\
\hline Porifera & & & & & & & & & & & & & & & & & & & & & & & & & & & & \\
\hline Halichondria panicea & $\mathrm{x}$ & $\mathrm{x}$ & & & & & & & & & & & & & & & & & & & & & & & & & & \\
\hline Halichondria sitiens & $\mathrm{x}$ & & & & & & & & & & & & $\mathrm{x}$ & & & & & & & & & & & & & & & \\
\hline Haliclona aquaeductus & & & & & & & & & & & & & $\mathrm{x}$ & & & & & & & & & & & & & & & \\
\hline Hymeniacidon assimilis & $\mathrm{x}$ & $\mathrm{x}$ & & & & & & & & & & & & $\mathrm{x}$ & $\mathrm{x}$ & & & & & & & & $\mathrm{x}$ & & & & & \\
\hline Porifera sp. 1 & & & & & & $\mathrm{x}$ & & & & & & & & & & & & & & & & & & & & & & \\
\hline Porifera sp. 2 & & & $\mathrm{x}$ & & & & & & & & & & & & & & & & & & & & & & & & & \\
\hline Porifera sp. 3 & & & & & & $\mathrm{x}$ & & & & & & & & & & & & & & & & & & & & & & \\
\hline Suberites ficus & & & & & & & & & & & & & & & $\mathrm{x}$ & & & & & & & & & & & & & \\
\hline Brachiopoda & & & & & & $\mathrm{x}$ & & & & & & & & & & & & & & & & & & & & & & \\
\hline Bryozoa & & & & & & & & & & & & & & & & & & & & & & & & & & & & \\
\hline Alcyonidium gelatinosum & $\mathrm{x}$ & $\mathrm{x}$ & $\mathrm{x}$ & & & $\mathrm{x}$ & & & & & & & & & $\mathrm{x}$ & & & & & & & $\mathrm{x}$ & $\mathrm{x}$ & & & & & \\
\hline $\begin{array}{l}\text { Bowerbankia composita \& } \\
\text { Patinella sp. }\end{array}$ & & $\mathrm{x}$ & & & & & & & & & $\mathrm{x}$ & & & & $\mathrm{x}$ & & & & & & & $\mathrm{x}$ & & & & & & \\
\hline Bryozoa sp. 1 & & & & & & $\mathrm{x}$ & & & & & & & & & & & & & & & & & & & & & & \\
\hline Bryozoa sp. 2 & & & & & & & & & & & & & & & $\mathrm{x}$ & & & & & & & & & & & & & \\
\hline Bryozoa sp. 3 & & & $\mathrm{x}$ & & & & & & & & & & & & & & & & & & & & & & & & & \\
\hline Cauloramphus sp. & & & & & & & & & & & & & & & & & & & & & & $\mathrm{x}$ & & & & & & \\
\hline Cellepora sp. & & & & & & & & & & & & & & & & & & & & & & $\mathrm{x}$ & & & & & & \\
\hline Dendrobeania cf. fruticosa & $\mathrm{x}$ & & & & & $\mathrm{x}$ & & & & & & & & & & & & & & & & & & & & & & \\
\hline Dendrobeania levinseni & & & & & & & & & & & & & & & & & & & & & & & $\mathrm{x}$ & & & & & \\
\hline Eucratea loricata & & $\mathrm{x}$ & & & & $\mathrm{x}$ & & & & & & & & & & & & & & & & & & & & & $\mathrm{x}$ & \\
\hline Flustra nordenskjoldi & $\mathrm{x}$ & & & & & $\mathrm{x}$ & & & & & & & & $\mathrm{x}$ & & & & & & & & & $\mathrm{x}$ & & & & & \\
\hline Flustra serratula & & & & & & $\mathrm{x}$ & & & & & & & & & & & & & & & & & & & & & & \\
\hline $\begin{array}{l}\text { Myriapora subgracilis \& } \\
\text { M. coarctata }\end{array}$ & & & & & & $\mathrm{x}$ & & & & & & & $\mathrm{x}$ & & & & & & & & & & & & & & & \\
\hline Porella beringia & & & & & & & & & & & & & & & & & & & & & & $\mathrm{x}$ & & & & & $\mathrm{x}$ & \\
\hline Porella compressa \& Tegella sp. & $\mathrm{x}$ & & & & & & & & & & & & $\mathrm{x}$ & & & & & & & & & $\mathrm{x}$ & & & & & & \\
\hline Porella saccata & & & & & & & & & & & & & $\mathrm{x}$ & & & & & & & & & & & & & & & \\
\hline Rhamphostomella sp. & & & & & & & & & & & & & $\mathrm{x}$ & & & & & & & & & & & & & & & \\
\hline Sarsiflustra securifrons & & & & & & $\mathrm{x}$ & & & & & & & & & & & & & & & & & & & & & & \\
\hline Semibugula birulai & & & & & & & & & & & & & & & & & & & & & & $\mathrm{x}$ & & & & & & \\
\hline
\end{tabular}

